\title{
LIST OF THE MEMBERS
}

OF THE

\section{ROYAL ASIATIC SOCIETY}

or

\section{GREAT BRITAIN AND IRELAND}

FOUNDED MARCH, 1823

CORRECTED TO 20TH JUNE, 1922

74 GROSVENOR S'TREET •

LONDON, W. 1 


\section{ROYAL ASIA'IIC SOCIEI'Y}

\section{Patron}

HIS MOST EXCELLENT MAJESTY THE KING.

\section{Vice-Patrons}

HIS ROYAL HIGHNESS THE PRINCE OF WALES.

FIELD-MARSHAL HIS ROYAL HIGHNESS THE DUKE OF CONNAUGHT.

THE VICEROY OF INDIA.

THE SECRETARY OF STATE FOR INDIA.

\section{Honorary Vice-Presidents}

1919 REV. A. H. SAYCE, D.LITT., LL.D., D.D.

1922 LIEUT.-COL. SIR RICHARD C. TEMPLE, BART., C.B., C.I.E., F.S.A.

\section{COUNCIL OF MANAGEMENT FOR 1922-23}

\section{President}

1922 THE RIGHT HON. LORD CHALMERS, G.C.B., ETC.

\section{Vice-Presidents}

1922 PROFESSOR E. G. BROWNE, M.A., F.B.A.

1921 M. GASTER EsQ., Рн.D.

1920 SIR GEORGE A. GRIERSON, K.C.I.E., Ph.D., D.LitT., LL.D., ETc.

1920 SIR HENRY H. HOWORTH, K.C.I.E., D.C.L., F.R.S.

1921 PROFESSOR A. A. MACDONELL, M.A., PH.D., F.B.A.

\section{Honorary Officers}

1921 F. W. THOMAS, Esq., M.A., PH.D. (Hon. Secretary).

1922 H. B. MORSE, Ese., LL. D. (Hon. Treasurer).

1921 A. G. ELLIS, EsQ., M.A. (Hon. Librarian).

1921

1922

1920

1922

1922

1919

1921

1922

1921

1919

1921

1919

1921

1919

1919

1921

\section{Ordinary Members of Council}

\section{REV. T. GRAHAME BAILEY, D.LTTT., B.D., M.A.}

PROFESSOR L. D. BARNETT, M.A., LiTT. D.

C. O. BLAGDEN, Esq., M.A., LL.D.

R. GRANT BROWN, EsQ., I.C.S. (ret.).

A. E. COWLEY, EsQ., M.A., D.LITt.

W. FOSTER, EsQ., C.I.E.

I. GILES, Esq., M.A., D.LitT.

H. R. HALL, EsQ., D.LITT., F.S.A.

PROFESSOR S. H. LANGDON, M.A., Ph.D.

PROFESSOR D. S. MARGOLIOUTH, M.A., F.B.A., LITT.D.

F. E. PARGITER, EsQ., M.A., I.C.S. (ret.).

E. S. M. PEROWNE, EsQ., F.S.A.

SIR E. DENISON ROSS, KT., C.I.E., PH.D.

W. H. RYIANDS, ESQ., F.S.A.

R. CAMPBELL THOMPSON, Esq., M.A., F.S.A.

W. PERCEVAL YETTS, Esq., O.B.E.

\section{Secretary}

1920 MISS ELLA SYKES.

\section{Asst. Secretary}

1917 MISS L. B. PHILLIPS.

\section{Librarian}

1919 MISS F. H. LATIMER.

\section{Honorary Solicitor}

ALEXANDER HAYMAN WILSON, ESQ.,

Westminster Chambers, 5 Victoria Street, S.W. 1. 


\section{COMMITTEES}

\section{Finance and Publications}

DR. M. GASTER.

SIR GEORGE GRIERSON.

SIR HENRY HOWORTH.

PROF. A. A. MACDONELL.
PROF. D. S. MARGOLIOUTH.

MR. E. S. M. PEROWNE.

MR. PERCEVAL YETTS.

\section{Library}

DR. I. D. BARNETT.

MR. WM. FOSTER.

PROF. S. LANGDON.

MR. R. SEWELL.

\section{Entente}

MR. C. OTTO BLAGDEN.

MR. F. E. PARGITER.

DR. A. E. C. COWLEY.

PROF. E. J. RAPSON.

SIR GEORGE GRIERSON.

\section{Forlong Fund}

MR. C. O. BLAGDEN.

MR. E. S. M. PEROWNE.

DR. H. R. HALL.

SIR E. DENISON ROSS.

PROF. A. A. MACDONELL.

MR. W. PERCEVAL YETTS.

\section{Public Schools Gold Medal}

MR. R. GRANT BROWN.

MR. WM. FOSTER.

DR. M. GASTER.

PROF. D. S. MARGOLIOUTH.
MR. F. E. PARGITER. PROF. E. J. RAPSON. SIR E. DENISON ROSS.

\section{Honorary Auditors, 1922-23}

MRS. FRAZER (for the Society).

MR. L. C. HOPKINS (for the Council).

* The President of the Society and the Honorary Officers of the Society are ex-officio members of all Committees. 


\section{SIIDemberg}

\section{RESIDENT AND NON-RESIDENT}

N.B.-The marks prefixed to the names signify-

* Non-resident Members.

$\dagger$ Members who have compounded for their subscriptions.

$\ddagger$ Library Members.

$\S$ Members who have served on the Council.

1902 His Most Excellent Majesty the Kiva, K.G.

1921 His Royal Highness the Prince of Wales, K.G.

1882 Field-Marshal His Royal Highness the Duke of Connaught, K.G.

1920 *ABD-EL-RAZEK, Sheikb, M.H., School of Oriental Studies, Finsbury Circus, E.C. 2.

1921 *AmRaham, Capt. R. C., c/a Hong Kong and Shanghai Bank, Bombay.

1919 *A brahams, Israel, M.A., D.D., Christ's College; 11 St. Barnabas Rd., Cambridge.

1917 *adhikari, N. S., Gandevi, Surat, Bombay.

1919 *AvIL, Maulvi Mohd. Akhtar, M.A., L.L.B., Head Master, Govt. High School, Bareilly, U.P.

1921 *AFzal, Ali, Diwan of Nawab Bahadur of Murshidabad, Bengal.

101912 *AFZaL, Nawabzada Bahadur Khwaja Muhammad, The Palace, 1 Armenian St., Dacca, E.B. \& A.

1900 *AmMad, Aziz-uddin, Khan Bahadur, Judicial Minister, Dholpur, Rajputana.

1918 *Afmad, Fakhruddin, M.A., Hastings House, Alipur, Calcutta.

1919 *Ammad, Khwaja Amir, Ansari, B.A., Mohalla Ansar, Panipat, 1slamia High School, Lucknow, U.P.

1921 *Ammad, Khwaja Latif, B.A., Govt. Mohammadan High School, Amraoti Camp, Berar.

1909

*Afmad, Maulavi Kamaluddin, Shams ul-Ulama, M.A., c/o King, Hamilton \& Co., Calculta.

1921 *Aнmed, S. A., F.R.S.A., 55 Modhu Sudhan Biswas' Lane, Howrah.

1922 *Ahmed TaWaduddin, M.A., Dy. Magistrate and Dy. Collector, Julpaiguri, Bengal.

1912 *ArsscoUgh, T. M., O.B.E., H.M. Senior Trade Commissioner, Allahabad Bank Buildings, 6 Royal Exchange Place, Calcutta.

1903 *Aryangar, Prof. S. Krishnaswami, Univ. of Madras, Sri Venkatesa Vilas, Nadu St., Mylapore, Madras.

201921 *AtYaR, B. V. Kameswara, M.A., Pudukstah, S. India.

1906 *AIYAR, K. G. Sesha, High Court Judge, Trivandrum, Travancore.

1915 *Aryar, V. Natesa, B.A., Supt. Arch. Suivey, Frontier Circle, Peshawar, N.W.F.P. 
1916

1912

1917

1917

1919

I920

1922

$30 \quad 1921$

1921

1907

1909

1922

1909

1904

1904

1919

1921

$40 \quad 1915$

1922

1921
1921

1922

1921

1922

1918

1917

1920

$50 \quad 1903$

1918

1919

1917

1921

1921

*Atyengar, A. R. Duraswami, 2/8 Lakshmi Vilas, Sunkuvar Agraharam St., Chintadripet, Madras.

*ArYer, Kandadai V. Subramanya, B.A., Asst. Supt. for Epigraphy, Ootacamund, Madras.

*Aryer, M. S. Ramaswami, B.A., Beach House, Mylapore, Madras.

*Aryer, Padmanabha R., B.A., Taikad, Trivandrum, Travancore.

*Alam, Sahibzada Mohammed Habib, 1 Prince Anwar Shah Rd., Tallygunge, Calcutta.

*Albetght, Wm. F., Ph.D., American School of Oriental Research, Box 333, Jerusalem.

*AuI, A. F. M. Abdul, Asst. Sec. to Govt. of India, Imperial Record Dept., Calcutta.

*Alr, Mir Anwar, P.C.S;, Anver Cottage, Saifabad, Hyderabad, Deccan.

*Aur, Md. Zafr, M.A., Qazi, Edwardes College, Peshawar, N.W.P.

*ALI, Muhammad Azhare, Etimadpur, Agra, U.P.

*Alı, Saiyid Aijaz, Dep. Collector, P.O. Nadbai, Bharatpur Stale, Rajputana.

*AlI, S. Barkat, Quaraishi, Chief Mauivi Board of Examiners, 1st Council House Street, Calcutta.

Allay, J., M.A., British Museum, W.C. 1.

*Alvarmz, Justin C. W., I.S.O., H.B.M. Consul, Tripoli; Union Club, Malta.

§Ametr Au, Rt. Hon. Syed, C.I.E., LL.D., 2 Cadogan Place, S.W. 1.

*Amuнad, V., Château du Boucheron, Bosnie, Hte. Vienne, France.

AnDERson, Frederick, 54 Queen's Gate, S.W. 7.

*Antanr, Pandit Rati Lal M., B.A., A ppellate Judge, Jhalawar State, Jhalrapatan, Rajputana.

*Anwar-dL-HAQQ, Zia-ul-Ulum, M.A , Mufti, Munshi-Farzil, Director of Pub. Instruction, Bhopal State.

†*Armbruster, Maj. Chas. H., M.A., O.B.E., Sinkat S.C.S., Sudan.

*Ayscodgh, Mrs. F., c/o Scott, Harding \& Co., 6 Pekin Road, Shanghai, China.

*Ayyar, R. Satyamurti, M.A., M.L., Munsif, Tiruvalur, Tanjore.

*Azar, Prof. Sirajuddin, M.A., Islamia Coll., Lahore, Panjab.

*Azrz, Abdul, Mir Munshi, Old Fort, Jullunder City, Panjab.

*BA, Saya Maung, Head Master, A.B. High School, Ahlone, Rangoon, Burma.

Baddetey, J. T., 34 Bruton St., W. 1.

*BagChI, Phanibhusan, B.A., Vidgabhusan Zamindar, 9 Nithak, P.O. Kakina, Rangpur, N. Bengal.

§BarleY, Rev. T. Grahame, D.Litt., B.D., M.A., Sch. of Oriental Studies, Finsbury Circus, E.C. 2.

*Bakng, Lt. Chas. C., Box 296, Lancaster, Cal., U.S.A.

*Batc, Chas. Edw., 52 Croydon Grove, W. Croydon.

*Bamandasji, Ragvaid Sri, Kaviraj, 152 Harrison Rd., Calcutta.

*Bamber, Chas. A., 32 Stanley Av., Sefton Pk., Bristol.

*Banerjea, Babu A. C., Pleader, 3 Socrah 1st Lane, Beliaghatta, Calcutta. 
1910 *BangrJea, Dr. Rasbihari, M.B., F.I.A.Sc., Santi Cootir, Bally, Hourah, Bengal.

1922 *BanerJte, Dr. B. K., M.B., P. Office, Dhakuria, S. Calcutta.

1917 *Banerufe, Babu Kedareswar, B.A., Asst. Engineer, P.O. Nabadwip, Bengal.

1921 *BanhrJ Ee, Babu Satya Kishore, M.A., Zamindar, 85 Up. Circular Rd., Calcutta.

601921 *Bantru, A. K., M.A., B.L., Pleader, Judge's Ct., Alipur, 106/4 Amherst St., Calcutta.

1921 *BanerJI, A. P., M.A., Sasiri, 30 Warwick St., Oxford.

1920 *Banerji, Dr. Debi Pada, M.B., The Santi Pharmacy, 133 Up. Chitpur Rd., Calcutta.

1920 *BanerJI, P. N., 57 Chakrabw Road North, Bhawanipur, Calcutta.

1919 *BanerJi, R. N., B.Sc., Phys.-in-Charge, Chemical Laborxtory, 13 Edmonstone Rd., Allahabad.

1918 *Baraket-Uli.ah, M.A., Prof. of Philosophy, Forman Christian College, Lahore.

1912 *Barnard, J. T. O., C.I.E., Asst. Commiseioner, Myit Kyina, Burma.

1904 §Barnett, Lionel D., Litt.D., Prof. of Sanskrit, University College; British Museum, W.C. 1.

$1890 * \dagger$ Baroda, H.H. Sir Sayaji Rao Gaekwar Bahadur, G.C.S.I., G.C.I.E;, Maharaja of.

1921 *BASAK, Radhagovinda, M.A., Lecturer Dacca Univ., 3-4 Mahajanpur Lane, Dacca.

70 Hon. 1906 Basset, Réné, Prof. of Arabic, Villa Louise, Rue Denfort, Rochereau, Algiers.

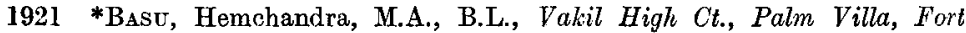
Monghyr, Calcutta.

1920 *Basv, Praphullachandra, Prof. of Economics, Holkar College, Indore.

1922 *Basu, Babu Upendra Mohan, c/o Rai P. Mohan Basu, Dy. Collector and Magistrate, Mymensingh, Bengal.

1881 *†Batw, Rev. J. Drew, $23 \notin$ Portsdown Rd., W. 9.

1909 *Bavanandam Pillat, S., Diwan Bahadur, Ananda Baugh, Vepery, Madras.

1907 *Brazley, Prof. C. Raymond, D.Litt., The University, Edmund St., Birmingham.

1919 BeLI, George, 55 Walpole St., Kev, Victoria, Australia.

1901 BecL, Miss Gertrude, 95 Sloane St., S.W. 1.

1921 *Ber.shaH, Haji A. Mejid, Sch. of Oriental Studies, Finsbury Circus, E.C.2.

801913 *†Belvalkar, Shripad K., M.A., Ph.D., Prof. of Sanskrt, Deccan College, Bilvakunja, Bhamburda, Poona.

1913 *Bernard, Pierre A., Shastri, Box 27, 662 Wes: End Av., New York, U.S.A.

1892 *Bevan, A. A., M.A., Lord Almoner's Realer in Arabic, Trinity College, Cambridge.

1893 §Beveridae, H., 53 Campden House Road, W. 8.

1899 \$Beveridge, Mrs. H., 53 Campden House Road, W. 8.

1882 *†BнAвba, Rev. Shapurje D., M.D., 8 Drakefell Rd., S.E. 14. 
1920 *BhandaRe, Sadānand T., J.P., F.S.A., Rao Bahadur, 410 Thakurduar, Post No. 2, Bombay.

1921 *Bhandari, M. Chand, Chief Sec. to Rana Sahib of Kunihar State, Simla Hills.

How. 1885 BhandarkaR, Sir Ramkrishna Gopal, K.C.I.E., LL.D., Sangamaśrama, Poona, Bombay.

1920 *BharadwaJa, Prof. Yajnavalkya, M.Sc., St. John's College, Agra, U.P.

901920 *Bhardvaj, Dattatreya K., B.A., Vidyabhusan, Prof. of Ind. Hist. and Sanskrit, E1. Tilal S indesh, Mangalore, S. India.

1918 *Bhat, V. G., B.A.

1922

*Bhatnagar, Raghubar D., Sen. Sub. Dy. Inspector of Schools, Disi. Hamirpur, U.P.

1921

1909

*Bhattachary s, Benoytosh, M.A., 26 Pataldanga St., Calcutia.

1912

*Bhattacharya, Babu Bisiswar, Dep. Magistrate, 4 Madhan Chatterjea's 2nd Lane, Elgin Road P.O., Calcutta.

*Bhattacharyya, Babu Jyotish Chandra, M.A., Vidyalankar, Vakil High Court, Purnea, Bihar, India.

1421

*Bhushan, Bibbuti, Rai Chandhuri, 20 Formosa St., W. 9.

1919

1921

*BIswas, Nagendranath, B.A., The Brahmacharya Vidyalaya, Ranchi, $B . \& O$.

1911

*Bivar, H. G. S., c/o Grindlay \& Co., 11 Hastings Street, Calculta.

100

*Blackman, A. M., Litt.D., 24 Bardwell Rd., Oxford.

1895 §Bragdev, C. Otto, 57 Earl's Court Sq., S.W. 5.

1909

1922

1921

*Blunt, E. A. H., I.C.S., clo Secretariat, Allahabad, U.P.

1921

*Bobrnskoy, Count Alexis, B.A., clo H.S. King \& Co., 65 Cornhill, E.C.2.

1922 *Bondccr, Prof. Alessandro, Univ. of Siena, 2 via Baldeschi, Perugia,

*BonnerJee, Rai Sahib Pandit G. L., B.A., Kaviratna Board of Examiners, 11 Patuatola Lane, Calcutta. Italy.

1920

*Bowra, Cecil A. V., Chief Sec., Inspectorate Gen. of Customs, Peking, China.

1921

*Bowstrad, J., Ranchi, Chola Nagmur.

1911

1921

*Boyer, M. l'Abbé A. M., 114 Rue du Bac, Paris, VIIe.

1907 §Rown, R. Grant, I.C.S. (ret.), Hon. Treasurer, East India United Service Club, St. James's Sq., S.W. 1.

$1101921 *^{*}$ Brown, S. M., D.F.C.

1917 Brown, Thos., La Roque, Overton Road, Sutton, Surrey.

1889*†BRowne, Edw. G., M.A., F.B.A., Adams Prof. of Arabic; Pembroke College ; Firuood, Trumpington Rd., Cambridge.

1919 Brownen, G., F.C.S., Christchurch, Hants.

1922 BRUCE-Low, Robert, Ministry of Heallh, S.W. 1.

1907 *BRunton, Major Chisholm D., 6 Learmouth Place, Edinburgh.

1908 *Büchler, Dr. A., Jews' Coll.; 261 Goldhurst Terrace, N.W.6.

1919 Bddge, Sir E. A. Wallis, Litt.D., F.S.A., British Museum, W.C. 1.

$1906 * \dagger$ * Burdwan, Hon. Maharajadhiraj Sir Bijay Chand M. Bahadur, Maharaja of, 1.O.M., K.C.S.I., K.C.I.E., The Palace, Burdwan, Bengal.

1921 Buchra, Shapurji N., B.E., Shakespeare Hut, Keppel St., W.C. 1,

Bunkitт, Prof. F. C., D.D., West Road Corner, Cambridge. 
1897 *Burn, Richard, C.S.I., c/o Grindlay \& Co., 54 Parliament St., S.W. 1.

1918 *Burrowas, Major H. O. A. D., c/o Melbourne C. of E. Gram. School, Domain Rd., S. Yarra, Victoria, Australia.

HoN. 1920 Caland, Prof. W., Koningslaan 78, Utrecht, Holland.

1921 *CampBeLL, J. S. F., Christ Church College, Oxford.

1921 *Cardemlac, Pierre, Directeur de la Banque Russo-Asiati?ue, 2 rue du Pradeau, Tarbes, Htes. Pyrenées, France.

1921 *Carmtchael, Wm. B. G., Balliol College, Oxfori.

1890 *Carpenter, Rev. J. Estlin, D.Litt., 11 Marston Ferry Rd., Oxford.

1919 CARR, R. C. Culling, O.B.E., I.C.S. (ret.), 5 Fitzjames $A v ., W .14$.

1888 Hon. 1919 Casartelli, Rt. Rev. L. C., Bishop of Salford, St. Bede's College, Manchester.

1301921 *Chakladar, H. C., M.A., Lecturer in Ancient Ind. Hist., Univ. of Calcutta, 25 Sahangar Rd., Kalighab, Calcutta.

1917 *Chakravart, Phani B., B.A., Sub-Dep. Magistrate 1st Class, Hourrah, Bengal.

1899 *Chakravarti, Rai Bahadur M. Mohun, M.A., 14 Palmer Bazar Rd., P.O. N. Intally, Calcutta.

1921 *Chakpavartx, Satis Chandra, Zemindar, Dholla P.O., Mymensingh, Bengal.

1922 Chalmers, Lord, G.C.B., P.C., President, 3 Cornwall Mansions, Kensington Court, W. 8.

1877 *Chamberlain, Basil Hall, Hôtel Richmond, Geneva, Switzerland.

1895 *†Chand, Dewan Tek, O.B.E., I.C.S., Commissioner, Ambala Division, Ambala Cantt., Panjab.

1921 *Chand, Tara, M.A., Prof. of History, Kayastha Pathshala College, c/o National Provincial \& Union Bank of England, Oxford.

1919 *Chanda, Prof. Rama Prasad, 37a Police Hospital Rd., Intally P.O., Calcutta.

1919 *Chandra, Ram, B.A., LI.B., Prof. Gurukul Univ., 14/41 Orderly Bazar, Benares Coutt, U.P.

1401919 *Chandra, Ram, M.A., M.B.F., Brookhurst Lodge, Simla.

1920 *Charax, Shyama, Rai, B.A., LL.B., Pleader, Chhindwara, O.P.

1920 *Charpentier, Jarl, Ph.D., Götgatan 12, Upsala, Sweden.

1914 * †Chathoorbhoojadass, Dewan Bahadur Govindass, 459 Mint St., George Town, Madras, E.C.

1912 *Chatterjea, Babu Arun Chandra, Medical Practitioncr, Nabadwip, Nadia, Bengal.

1920 *ChatterJea, Basanta K., Insp, of Post Offices, Dhanabad, Manthoom, $B$. \& $O$.

1915 *ChatterJee, Hon. Mr. M. Atul Chandra, I.C.S., Board of Industrirs \& Munitions, Simla.

1921 *Chat'ferJen, Rai Sahib N. C., Supt. Board of Examiners, 1 Council House St., Calcutta.

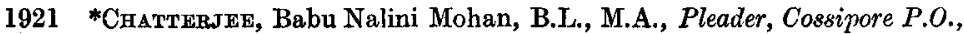
Tala, Calcutta.

1921 *ChatTerJee, Nirmal Chandra, M.A., B.L., Lecturer Calcutta Univ., 52 Haris Mukerji Road, Calcutta. 
$1501921{ }^{*}$ Chatterjee, Sadhou P., c/o B. Murray \& Co., 30 Clive St., Calcutta.

1919 *Chaturvent, Pandit Gulzari Lal, Rais, Kaimganj, Farrukhabad, U.P.

1915 *Chaturvedy, Pandit Shiv Kumar, B.A., Sec., Jhalawar State Council, Jhalrapatan, Rajputana.

$1914{ }^{*}$ Chaddhurr, Charu Ch., Rai Bahadur, Zamindar \& Hon. Magistrate, Sherpur Town P.O., Mymensingh, Bengal.

1915 *Chaddhori, Babu Hemanga Ch., Zamindar \& Hon. Magistrate, Sherpur Town P.O., Mymensingh, Bengal.

1914 *Chaudhuri, Babu Gopaldas, Zamindar, 32 Beadon Row, Caleutta.

1916 *Chaddhuri, Umes Ch. Sinha, Asst. Master Dutt H.E. School, Netrakona P.O., Mymensingh, Bengal.

1922 *Cha.dohury, D. Nath Ray, I.D.S.M., B.A., 161 в Balulbagan Road, Calcutta.

1915 *Chaudhury, Babu Hirankumar Roy, Zamindar, Basanta, Barisal, Bengal.

1921 *CHemma, M. S., B.A., 9 Walton Streei, Oxford.

1601921

Chelmsford, Viscount, G.C.M.G., G.C.S.I., G.C.I.E., G.B.E., 116 Eaton Square, S.W. 1.

1921 * Сh тетт, O. A. O. K. Chidambaram, Banker, Pallatur, S. India.

1918 *Chrzen-Akamuna, Shin-shu Otani, Daigaku, Kuramaguchi, Kyoto, Japan.

$1914{ }^{*}$ ChоцMelex, N. G., C.S.I., I.C.S. (ret.), Uphill, Bude, Cornwall.

$1920{ }^{*}$ Chopra, R. L., M.A., 11 George Sq., Edinburgh.

1921 *Chowdhuri, Dr. P. Nath, Ghazipore, U.P.

1922 *Chowdhury, K. K., L.M.P., The Toun Medical Hall, Pegu, Burma.

$1919 *^{*}$ ClaRK, Dr. Walter E., Prof. of Sanskrit, Univ. of Chicago, Ill., U.S.A.

1912 Clauson, Capt. Gerard L. M., O.B.E., 39a Clanricarde Gdns., W. 2.

1904 *Cuementr, C., Colonial Secretariat, Georgetown, British Guiana.

170 Hon. 1920 Clermont-Ganneau, Prof. Ch., Membre de l'Institut, 1 Av. de l'Alma, Paris, XIVe.

1911 *Clifton, Rev. Edw. Jas., O.B.F., Hon. C.F.," Sandown," Chorleywood, West Herts.

$1907{ }^{*}$ † Соснім, H.H. Maharaja Sri Sir Rama Varma, G.C.I.E., of, S. India.

1907 *Cochran, Alexander Smith, Yonkers, N.Y., U.S.A.

1910 * Codrington, Humphrey W., c/o Secretariat, Colombo, Ceylon.

1920 *CoEDÈs, George, Librarian Vajirañāna Nat. Library, Bangkok, Siam.

$1909{ }^{*}$ Cohen, Samuel J., 11 Peter St., Manchester.

1908 Colnstream, W., I.C.S. (ret.), 69 W. Cromwell Rd., S.W. 5.

1912 *Collins, Godfrey F. S., I.C.S., c/o Grindlay \& Co., Bombay.

1920 Coок, S. A., Litt.D., 26 Lensfield Rd., Cambridge.

1801919 Cooke, Richard, The Croft, Detling, Maidstone.

1906 *†Coomaraswamy, A. K., D.Sc., Keeper of Indian Art, Museum of Fine Arts, Boston, Mass., U.S.A.

HoN. 1893 Condran, Prof. Henri, 8 Rue de Siam, Paris, XVIe.

1821 *Cotterinl, W. S., Govt. Officer, Treasury, Miri, Sarawak, via Singapore.

1888 Couswes, Henry, c/o Grindlay \& Co., 54 Parliament St., S.W. 1.

1919 Cousland, Mrs. (Gertrude), 17 Cholmeley Crescent, N. 6.

1915 *§Cowlex, A. F., M.A., D.Litt., Bodley's Librarian; Magdalen College; 94 St. Aldate's, Oxford. 
1919 *Crawford, Samuel J., B.A., B.Litt., Prof. of English Philology, Christian College, Madras.

$1912{ }^{*}$ Creswetl, Capt. K. A. C., 12 Regant's Park Rd., N.W. 1.

1919 Crum, W. E., 13 Cavendish Rd., Westbury-on-Trym, Bristol.

1901915 Cumming, Sir John Ghest, K.C.I.E., C.S.I., 83 Hodford Rd.; N.W. 11.

1909 Curzon of Kfadeston, Marquess, K.G., G.C.S.I., G.C.I.E., F.R.S., 1 Carlton House Terrace, S.W. 1.

1908 *Daiches, Dr. Samuel, Prof. Jews' College, Guildford St., W.C. 1.

1891 *†D'Alviel. A, Comte Goblet, Palais des Académies, Brussels.

1921 *Danby, Rev. Herbert, St. George's Cathedral, Jerusalem.

1909 *Dandoy, Rev. G., S.J., St. Mary's, Kurseong.

1921 *DaR, Mukat B. Lal, B.Sc., LL.B., Dep. Collector, Banda, U.P.

1921 *Darnvalca, Jehangir D., Waldorf Hotel, W.C. 2.

1922 *Das, Ajit Nath, 24A South Road, Enially, Calcutla.

2001921

1916

1921

1920

1922

1913

1915

1904

1915

1894

1921

1921

1922

1920

1919 *†Debbarman, Pyari Mohan, B.Sc., Botanical Survey India, R. Botanical Gdns., Botanical Gdns. P.O., Howrah, Bengal.

Hon. 1908 Delimzsch, Geheimrat Pro:. Dr. Friedrich, Sï lstr. 47II Leipzig, Saxony.

1921

1920 *Das, Jatindra N., B.A., Sch. of Oriental Studies, Finsbury Circus, E.C. 2. *Das-Gupta, Nibaranchandra, B.A., Asst. Revaluation Off., Dinajpur P.O., Bengal.

*Das-Gupta, S. N., M.A., Ph.D., Trinity College, Cambridye.

*Datta, Prof. Bhava, Shastri, Govt. College, Ajmer.

*Datтa, B. K., B.Se., Indrani Villa, Pauchgram, Bengal.

*Datta, Jnanendra Nath, Supt. of Post Offies, Hooghly Div., Howrah, Bengal.

*Davar, Amolak Raj, 4 Buc? ingham Court, $113 \alpha$ Ripon St., Calcutla.

*Davar, M. B., M.A., Ph.D., 89 Lamington Rd., Bombay.

*Davres, Rev. A. W., St. John's College, Agra, U.P.

*†Davies, Rev. T. Witton, B.A., Ph.D., D.D., Prof. of Semitic Languages, Univ. Coll. of North Wales, Bryn Haul, Bangor.

*Davis, C. Noel, M.D., Med.Off.Municipal Health Dept., Shanghai, China.

*Dayal, Raghabar, M.A., M.O.I., Principal Sanatana Dharma College, Lahore, Panjab.

*DE, Sushil Kumar, 30-1 Beadon Row, Calcutta.

Dfane, Lady (Harold), 72 O:erstran $l$ Mans., S.W. 11.

*Deb, Sri G. N., Tatwanidhi, M.B., D.M., Second Prince of Tekhali, Tekkali P.O., Ganjam, Madras.

*Deb, Kumar Harit K., Sorabazar, Rajbati, Calcutta.

ENT, Capt. R. B., clo Nat. Bank of India, 26 Bishopsgate, E.C. 2.

*Deo, Maharajkumar Sri Sudhansu S. Sing, Sonpur Feudatory State, P.O. Sonpur Raj, via Sambalpur.

*Destka-Chart, Diwan Bahadur T., High Court Vakil, Cantonment. Trichinopoly, Madras.

*Deussen, Prof. P., 39 Besseler-allee, Kiel, Germany.

*Deva, Prof. Rama, The Gurukula, Mahavidyala, Kangri, P.O. Shampur,

*Das, Babu Tarak Ch., P.O. Ujancharbazar, Ujanchar, Tippera, Bengal. Bijnor, U.P. 
1920 *Devonshire, Mrs. R. L., Maadi, nr. Cairo.

1904 *Dewhurst, Robert Paget, M.A., I.C.S., 106 Abingdon Rd., Oxforrl.

1915 *Dey, Kumud Lall, Hon. Magistrate, Barabazar, Chinsurah, Hooghly, Bengal.

1908 *Dhaninivat, Mom Chow, Talat Noi House, Bangkok, Siam.

1920 *Dhar, Babu Goknlnath, B.A., Librarian, Presidency Coll., Calcutla.

1914 Dickinson, Miss M. Lowes, Shottersley, Haslemere, Surrey.

1922 *Drchson, Percy L., Western House, The Park, Nottingham.

2301908 *+Din, Malik Muhammad, Gen. Manager, Estates of the Nawab, Karnal, Panjab.

1894

*D'Ordmanbug, Serge, Ph.D., Prof. of Sanskrit, The University, Petrograd, Russia.

1919 Donalmson, Rev. E., Pyworthy Rectory, Holsworthy, Devon.

1920 *Doniach, A. S., Wadham College, Oxford.

1910 *Drake-Brockman, D. I., I.C.S., c/o T. Cook \& Son, Bombay.

1920 *Driver, G. R., M.A., Magdalen College, Oxford.

1921 *Duвn, Pandit Kailas Narayan, B.A., LL.B., Chief Judge, Barwan,, Cent. India.

1917 *Durai, Samuel Abraham, B.A., Law College, Fort, Bombay.

1907 §Duk.AND, Rt. Hon. Sir H. Mortimer, G.C.M.G., K.C.S.I., Penmayne House, Rock, St. Minver; Cornu;all.

1917 *Dutr, Prof. Anakul C., College House, Bareilly, U.P.

2401922

1919

*Dutr, H. N., B.A., 11 Kailas Bose's Lane, Howrah, Bengal.

*Duts, Kamala P., M.A., B.I., Off. in Charge Revenue Dept., Tippera State, Agartala P.O., Hill Tippera.

1917

1921

*Dutr, Ialita Prasad, 181 Manititola St., Calcutta.

*Dotra, Pandit Brahma, Shastri, Prof. Arya Samaj School, Saraswati Nivasa, Gur-ki-Mandi, Ayra.

1921

1921

1905

1919

1919

1921

250

1905

1921

1897

1919

1919

1907

1904

1921

1921

1919

*Epers, Robert F., Christ Church, Oxford.

*Edwarns, Mrs. C., 309 West 91 st Street, New York, U.S.A.

*Edwands, E., Oriental Boolss d MSS. Dept., British Museum, W.C. I.

*Edwardes, S. M., I.C.S. (ret.), Meraldene, Chislehurst, Kent.

*Effennt, Sirdar Abdul G. K., M.A., 14 Race Course Road, Lahore.

ELaOod, Cyril L., 19 Greencroft Gdns, , N.W. 6.

*Elrot, Rt. Hon. Sir Charles, K.C.M.G., C.B., British Embassy, Tokyo, Japan.

*Elliot, Mrs. A. de Zoete, Whirlon, Leigh-on-Sea.

\$Eutus, Alex. George, M.A., How. Limariar, 32 Willow Rd., N.W. 3.

Fucis, Miss M. F., 15 Waterloo Square, Bognor, Sussex.

Eu.Y, Alex. Kirkpatrick, Very Rev. Dean of, The Deanery, Ely.

*Enthoven, R. E., C.I.E., I.C.S., Barlavington Manor, Petworth, Sussex.

Etringhausen, Maurice L., 29 Downside Crescent, N.W. 3.

*Evans, I. H. N., Perak Museum, Taiping, F. M. States.

*Evans, J. H. F., Wadham College, Oxford.

Ev, Ella, Lady, The Alexandra Club, 12 Grosvenor St., W. 1.

2601922 *Fairweather, Wallace C., 62 Saint Vincent St., Glasgow.

1902 Fanshawe, Herbert Chas,, C.S.I., 72 Philbeach Gdns., S.W. 5. 
1881 *†Fargues, J.

1880 * †FARĪDŨN-JANG, Bahadur, Nawab Sir, K.C.I.E., C.S.I., Sadr-ul-Mahäm to $H . E x$. H. the Nizam of Hyderabad, Deccan.

1921 *Farmer, Henry, 102 Byres Rd., Glasgow.

1916 *Farquhar, J. N., D.Litt., 5 Russell St., Calcutta.

1914 *Fatah, Moulvi Syed A., K.-i-H., Zamindar, Rangpur, Bengal.

1914 *Ferauson, John C., Ph.D., Peking, China.

1901 *Ferausson, J. C., I.C.S., cjo H. S. King \& Co., 9 Pall Mall, S.W. 1.

1907 *Ferrar, Major M. L., c/o Grindlay \& Co., Bombay.

1893 *Frnot, Louis, Directeur de l'École Française d'Extrême Orient, Hanö, Indo-China.

1921 *Frsh, Rev. Thrs., St. Edmund's House, Cambridge.

1909 §Foster, Wm., C.I.E., Registrar \& Supt. of Records, India Office, S.W. 1.

Hon. 1918 Fovcher, A., 286 Boul. Raspail, Paris, XIVe.

1921 *Frampton, H. J., I.C.S., 56 Hurst St., Iffey Rd., Oxford.

$1907{ }^{*}$ Frasen, Chas. I., Council Office, W. Montreal, Canada.

1916 Frazer, Sir J. G., 1 Brick Court, Middle Temple, E.C. 4.

1918 Frazer, Mrs. R. W., The Hollies, Balcombe, Sussex.

1919 tFreER, Maj. W. J., V.D., F.S.A., Stonygate, Leicester.

1921 *Frisby, Alfred, 5 South Road, Kendal, Westmorland.

1912 *Fulton, A. S., Oriental Books \& MSS. Dept., British Museum, W.C. 1.

1918 *FurlaNI, Giuseppe, Ph.D., LL.D., S. Lorenzo di Mossa, Italy.

1921 *FuRnivali, J. S., clo Scott \& Co., Rangoon, Burma.

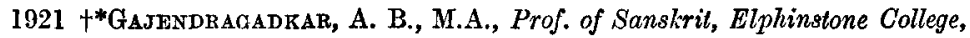
Bombay.

$1920{ }^{*}$ Ganlot, Sri Jagdiah Singh, Insp. of Forests \& Ed. “ Saini Shubhchintak", Gahlot House, Jodhpur.

1899 GaIT, Sir Edward A., K.C.S.I., C.I.E., The Croft, Park Hill, W. 5.

Hon. 1921 Ganapati Sissrē, T. Mahamahopadhyaya, Curator for Dept. for Publication of Sanskrit MSS., Trivandrum, Travancore.

$1919 *^{*}$ Ganester, T. S., M.A., Y.T., Maharaja's Collegiate S.hool, Mysore.

1916 *Ganaory, O. C., B.A., 12/1 Gangoly's Lane, Calcutta.

1912 *GaNaUly, Rabu Manomohan, District Engineer, 50 Raja Rajbullul's Street, Calcutta, India.

*GaRbeTT, Colin Campbell, C.I.E., I.C.S., c/o Grindlay \& Co., 54 Parliament St., S.W. 1.

1919 GaRdiner, Alan H., 9 Lansdowne Rd., W. 11.

$1915 *$ Garr, Banarsi Lal, B.Sc.

1890 §Gastrer, M., Ph.D., 193 Maida Vale, W. 9.

$1922 *^{*}$ G_TAM, Thakur L. S., B.A., Kavyatirtha, Tarighat, Ghazipore.

$1912 *^{*}$ Geden, Rev. A. S., Royapettah, Harpenden, Herts.

$1921{ }^{*}$ GeHror, Babu C. Bhuj, D.D.R., Supt. of Forests, near Gulab Sagar, Jodhpur, Rajputana.

$1906{ }^{*}+$ Gert, Wm. E., M.A., LL.D., Litt.D., Doylestoun, Pa., U.S.A.

$1919{ }^{*}$ Gerty, Miss Alice, 75 Av, des Champs Elysées, Paris.

$1921 *^{*}$ Ghatak, J. C., M.A., Prof. Diocesan Coll., 5 Baloram Bose Ghat Road, Bhawanipore P.O., Calcutta.

$3001921 *$ Gene, O. Thye, 39-2 Dickson Road, Singapore. 
1920 *Ghorpaday, Madhavrao H., 273 Somwar Peth, Anand Bhuran, Poona.

1920 *Ghosal, Haripada, Head Master, Raj H.E. School, P.O. Mahishadal, Midnapore, Bengal.

1918 *Grose, Nagendra Nath, B.A., Central Municipal Office, 5 Corporation Street, Calcutto.

1920 *Ghosh, Dr. Sarada C., F.C.P.S., 8 Sitaram Ghosh's St., Calcutta.

$1920{ }^{*}$ GноSн, Susil Kumar, B.A., 7 Ragendra Dutt Lane, Calcutta.

1908 *Ghosr, Wopendranath, Rai Bahadur, 13 Madhal Lane, Bhauranipur P.O., Calcutta.

1921 *Gibani, Sayid Aulad Ali, B.A., Oak Grove, Jharipani P.O., Dehra Dun, U.P.

1921 Grbbertson, Maj. G. W., 373 Holmesdale Rl., S.E. 25.

1921 *Gilder, Framroz N., Shakespeare Hut, Keppel Street, W.C.1.

3101919 §Grr.es, Lionel, M.A., D.Litt., Dept. of Oriental Books, British Museum, W.C. 1.

1912 *GIPPERICH, H., German Legation, Peling, China.

1916 *Goddard, Rev. W. G., M.S.A., Wesley Manse, S. Brisbane, Queensland, Australia.

How. 1893 Goldziner, Prof. Ignaz, 7 Holló-Utza, Budapest, Hungary.

1921 *Goodter, Most Rev. A., S.J., Arhbishop of Bombxy, Archbishop's House, Wodehouse Road Fort, Bombay.

1919 *Gopalamrishnan, S. S., Basel Mission College, Calicut.

1920 *GoptnatH, Pandit P., M.A., C.I.E., Rai Bxhadur, Member of state Council, Jaipur, Rajputana.

1884 * Gorparshad, Thakur, Talukdar of Baiswar, Aligarh.

1922 *Gourtay, W. R., Sec. to Governor of Bengal, Bath Club, Dover Street, W.1.

1910 *Graham, W. A., Adviser to Ministry of Agriculture, Bangkok, Siam.

3201919 †raves, R. E., 6 Grange Park, W. 5.

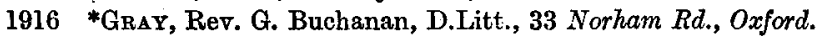

1919 Grane, F. W., Whitefield, Great shelford, Cambridge.

1918 Gremensiglds, Robert S., I.C.S. (ret.), 35 Clarges St., W. 1.

1893 *Greenup, Rev. Albert W., D.D., The Principal's Lodge, St. John's Hall, Highbury, N. 5.

1884 §Gringson, Sir George A., K.C.I.E., Ph.D., D.Litt., LL.D., F.B.A., I.C.S. (ret.), VICE-President, Rathfarnham, Camberley, Surrey.

1919 GrIfTITH, F. Ll., 11 Norham Gdns., Oxford.

1919 GRY, M. L., Recteur à l'Université, 10 Rue La Fontaine, Angers, N. et L. France.

1897 §Gudst, A. Rhuvon, 1a, Thornton Hill, S.W. 19.

Hon. 1898 GuIdr, Prof. Ignace, 24 Botteghe Oscure, Roma.

3301921 *Gurllaume, Rev. Prof. A., 43 North Bailey, Durham.

1910 *Gunawardiana, W. F., Dept. of Publiv Instruction, Rose Villa, Mt. Lavinia, Ceylon.

1921 *Gupta, B. L. Sen, B.A., 13-4 Ram Kanta Bose's 'Street, Baghbazar, Calcutta.

1920 *Gupta, Babu Kshetro M., M.B., Chief Med. Off., Mohishadal Raj, Bengal.

1915 *Gupta, Kumud Bandhu Das, B.A., 4th Presidency Magistrate, 2 Bankshah St., Calcutta.

1921 *Gupta, Pitam Lal, M.Sc., LL.B., Prof. of Mathematics, Raja Ram Colleye, Kolhapur. 
1913 *Guptd, Saurindra Kumar, B.A., B.L., B.Litt., Ph.D., Barr.-at-Law, Bar Library, Calcutta.

1919 *Gopta, Babu Shiva Prasad, Seva Upavana, Benares.

1894 *Gurdon, Lt.-Col. Philip R. T., C.S.I., I.A., c/o H. S. King \& Co., 9 Pall Mall, S.W. 1.

$1921{ }^{*}$ Gurner, Cyril W., I.C.S., c/o King, Hamilton \& Co., Calcutta.

1918 *Gunú, Kanhaya Lal, M.A., Inspector of Schools, Chattisgarh Circle, Raipur, C.P.

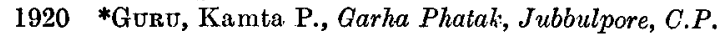

$1921{ }^{*}$ Gwrwn, R. M., M.A., Prof. of Hebrew, Trinity College, Dublin.

$1910{ }^{*} \mathrm{G}$, Maung Maung, Inspector of Excise, Opium Shop, Mandalay.

1921 *HABIв, M., B.A., Dali Bagh, Butler Rrad, Lucknou.

1902 *Hagopian, Prof. G., 25 Chesilton Rd., S.W. 6.

1920 *Hatdari, M. A. Khan, Akbar Manzil, Delhi.

1911 *HaIg, Kerest, Alloa, Seaford, Sussex.

$1898 *^{*}$ HaIt, Lt.-Col. Sir T. Wolseley, C.M.G., K.C.I.E., C.S.I., North Lodge, St. Leonards-on-Sea.

1919 *HaJela, Kripa Shankar, M.Sc., LL.B., Prof. of Mathematics, Raja Ram College, Kolhapore.

*Haldar, Babu Satya Charan, c/o Lale Babu K. Lal Haldae, Sub-Dy. Magistrate, Sandip, Noakali, Bengal.

1919 Hal., H. R., M.A., F.S.A., D.Litt., British Museum, W.C. 1.

1909 *Halliddy, Robert, Mount Pleasant, Moulmein, Burma.

1921 Hamid, Ungku Abdul, of Johore, 31 St. George's Rd., N.W. 6.

1920 *HandiquI, Krishna K., M.A., c/o Grindlay \& Co., 54 Parliament St., S.W. 1.

1904 *Hanson, Rev, O., Litt.D., Kachin Mission, Namlham, via Bhamo, Up. Burma.

$1920 * \mathrm{H}_{\Delta \mathrm{Q}}$, Prof. A., 116 Southmoor Rd., Oxford.

$1920{ }^{*} \mathrm{H}_{\mathrm{AQQ}}$, Prof. Qazi Fazl-i-, M.A., Govt. College, Lahore.

Hoy. 1921 Haraprasad Shastri, Mahamahopadhyaya, C.I.E., M.A., Prof. Univ. of Dacca.

1921 *Harder, Rev. T. M., LL.D., The Hostel, St. John's Hall, Highbury, N.5.

1915 *Hargreaves, H., Supt. of Archoeology, Lahore.

1910 *Harlex, A. M., M.A., Principal, Madrasah College, Calcutta.

1913 *HARPer, Rev. Robert, M.D., N. Shan States, Namkham, via Bhamo, Up. Burma.

1921 *Harris, Rev. E. N., American Baptist Paku Karen Mission, Toungoo, - Burma.

1919 HARRIS, Dr. Rendel, 172 Wellington Road, Whalley Range, Manchester.

1919 Harrison, Edgar E., 12 Leopold Rd., W. 5.

1919 Hartla nd, Ernest, Hardwick Court, Chepstow.

1920 *Hasan, A. N. M. Ali, M.A., Lechurer in Arabic \& Persian, Paina College, Bengal.

1919 *Hasan, Majid-ul-, c/o Y. H. Khan, Dep. Insp. Gen. of Police, Gosha Mahal, Hyderabad.

1920 *†Hassan-KhaN, Haji M. Ghulam, 4 Sadar Bazar Lines, Camp Karachi.

1921 HaY, George E., 96 Olive Rd., N.W. 2. 
1921 HaYashi, H. E. Baron, Japanese Ambassalor, 10 Grostenor Sq., W. 1.

1918 *Hayes, Rev. Herbert E. E., Missionary, C.M.S. House, Menouf, Egypt.

* Heming, Lieut.-CoI. Dempster.

1911 *Hratex, Prof. Johannes, Leisnigerstr. 24, Grossbauchlit: bei Döbeln, Saxony.

1912 *Hrturtcr, John, Minglands, Crumpsall Lane, Crumpsall, Manchester.

1901 *Hrul, Rev. J. R., S.P.G. Mission, Banda, U.P.

1913 Hilu, S. C., Ind. Educ. Serv. (ret.), 36 West Hexth Drive, N.W. 3.

$1885 \uparrow$ HrppISLEY, Alfred E., late Commissioner Chinese Customs, 8 Herbert Crescent, S.W. 1.

1891 *Hirschfeld, H., Ph.D., Lecturer on Semitics at Jews' Univ. Colleges; 14 Randolph Gdns., N.W. 6.

$380 \quad 1920$

1921

1919

1919

1918

1919

1915

1919

1911

1889 §Hopkins, Lionel Charles, I.S.O., The Garth, Haslemere.

3901908 Honnelt, Wm. Woodward, Ind. Edur. Serv., Office of Director of Public Instruction Bengal, Writers' Bldgs., Calcutta.

1919 *Hosain, Agher Hyder, A.D.C. to H.H. the Maharajah of Patiala.

Hon. 1902 Houtsma, Prof. M. T., Mahistraat 6, Utrecht, Holland.

1917 *Howarth, Major L. A., Political Agent, Muscat, Persian Gulf.

1919 §HoworTH, Sir Henry H., K.C.I.K., D.C.L., F.R.S., 45 Lexham Gdns., W.8.

1919 *Hочтема, D. van, Valeriusstraat, 92, Amsterdam.

1922 *HuQEe, Shah Syed Emdadul, M.L.C., Madagarganj P.O., Comilla, Tipperah, Bengal.

1921 *Hutron, J. H., C.I.E., I.C.S., Kohima, Naga Hills, Assam.

1908 *†Hyde, James H., Pavillon de l'Ermitage, 7 rue de l'Ermitage, Versailled, Seine et Oise, France.

1921 *Hrman, A., Wadham College, Oxford.

4001922 *Ikeda, Chotatsu, 21 Yamabushi-chō, Shitaya-ku, Tokyo, Japan.

1921 Inarams, Capt. Wm. H., Chake Chake, Pemba, Zanzibar.

1921 *Irwell, Mrs. H., 8 f Bickenhall Mans., W. 1.

1918 Ispahant, M. H., 21 Mincing Lane, E.C. 3.

1920 *Ivanow, W., c/o Asiatic Society of Bengat, 1 Park St., Calcutta.

1921 *Ives, C. St. John, Mailoor Estate, Kullakamby, Nilgiri Hills, S. India.

1920 *Iygngar, M. H. Krishna, Educ. Dept., Mysore.

1919 *Tyenaar, Prof. T. R. Sesha, 65 Coral Merchant St., Madras.

1916 *IYeR, K. Ramaswami, "New Home," Brahmins Club Road, Trivandrum, Travancore. 


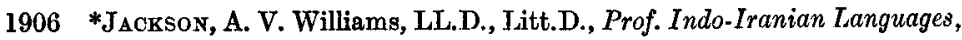
Columbia University, New York, U.S.A.

410 HoN. 1912 JАСовI, Dr. Hermann, Geb. Regierungsrat, Sanskrit Prof., 59 Niebuhrstr., Bonn, Germany.

1920 *JaGoe, Lt. H. E., K.A., 31 Mountain Battery, c/o Lucknow House, Outram Rd., Addiscombe, Surrey.

1922 *JaIN, Prof. S. P., M.A., Principal St. Stephen's College, Delhi.

1916 *JAINI, Jagmandir Lal, M.A., Bar.-at.Law, Saharanpur, U.P.

1901 *JARDINe, W. E., C.I.E., The Residency, Gwalior.

1920 *JaSHPUR, Jubraj Deo S. Singh, Deo'of, P.O. Jashpur, via Ranchi, C.P.

1883 *†JAXамонUN, Thakor Singh, Tahsildar of Seori Narayan, Bilaspur, C.P.

1922 *JAYASWAL, K. P., M.A., Mirzapur, U.P.

1918 *Jayatmaka, Don B., B.A., Advocate of Supreme Court Ceylon, Law Library, Colombo, Ceylon.

1920 *Jegferx, Rev. Arthur, M.A., American University,113 Kasr-el-Aini, Cairo, Egypt.

4201914 *JHa, Ganganatha, Prof. of Sanskrit, Sanskrit College, Benares.

1911 *†Jhalawar, H.H. Maharaj-Rana Sir Bhawani Singh, Bahadur, K.C.S.I. of, Jhalrapatan, Rajputana.

1882 *†Jinavaravansa, Rev. P. C., Buddhist Bhikshu (formerly Prince Prisdang), Dipaduttama Aräma, Koțahena, Colombo.

1921 *JoHN, Miss C. M.

1909 *Johnston, Edw. Hamilton, c/o Grindlay \& Co., Calcutta.

1904 *Jonnston, Reginald F., C.B.E., Chang Wang Hutung, Peling.

HoN. 1904 JoLIy, Prof. Julius, The University, Würzburg, Bavaria.

1919 Jones, H. Sefton, 18 Pembrole Gdns., W. 8.

1921 *Jones, Kenneth C., King Kothi Road, Hyderabad, Deccan.

1908 *Jopr, Chas. H. Keith, M.A., I.C.S. (ret.), Reader in Marathi, 16 Linton $R d .$, Oxford.

4301922 *JosHi, Pandit Ram Datta, B.A., Almora; U.P.

1911 *Jow ETr, Rev. Hardy, Govt. Offices, Weihaiwei, N. China.

JuDD, George H., F.R.C.S., 48 Chestnut Rd., S.E. 27.

1919 *Juna, Rabbi Leo, 10416 Columbia Av., Cleveland, O., U.S.A.

1918 *KaK, Ram Chandra, c/o Arch. Supt., Srinagar, Kashmir.

1917 *Kalia, D. R., M.B.E., Ferozpore, Panjab.

1920 *Kanshala, Prof. Ram S., Vidyabhusan, D.Litt., B.A., Ambala, Panjab.

1922 *KAUL, Pandit V. Nath, B.A., Naya Bazar, Gualior, C. India.

1917 *KÂvYanidHI, Babu Kali K., Vidyabinode, Parhatali Tillage, Mahamuni P.O., Chittagong, Bengal.

1909 *Karth, Alan Davidson, Prof. of English, Pangoon College, Burma.

1919 †KeITH, C. P., 308 Walnut St., Philadelphia, Pa., U.S.A.

1921 K.MP, Miss G. E., 26 Harley House, N.W. 1.

1895 *Kannedy, Miss Louise, Fairacre, Concord, Mass., U.S.A.

1909 *Kennedy, Pringle, M.A., c/o Grindlay \& Co., 54 Parliament St., S.W. 1.

1922 *Kennedy, Mrs. Wallace, 3 Florence Terrace, Londonderry.

1914 *Kent, A. S., c/o Chinese Post Office, Mukden, Manchuria.

1907 *Keonjhar, Raja Gopinath Narayan Bhunj Deo of, Orissa. 
1908 *Knsteven, Sir Chas. H., Royal Insurance Buildings, Dalhousie Square, Calcutta.

1921 *†Kharapur, H.H. Ali Namoz Khan Talpur, Mir of, G.C.I.E., Mirs Sind, India.

1916 *KHAN, Hon. Chaudhuri Moh. Ismail, Zamindar of Barisal, Bengal.

4501922 *KHax, Debendra Lal, M.L.C., Kumar of Naringole, Gope Palace, Midnapore, Bengal.

1921 *KHaN, J. S. Shahbaz, M.B.E., Bombay House, 2393 East Strect, Poona.

1911 *Khan, Mahomed Hasan, Khan Bahadur, Asst. Acct. General, $11 b$ Clive Rd., Allahabad, U.P.

1921 *Kran, Haji Malik S. Wali, Khan Bahadur, Rais \& Hon. Magistrate, Old City, Bareilly, U.P.

1921 *KHan, The Imam Mustapha, The Mosque, Woking.

1909 *Knncato, C. A., C.V.O., I.C.S., Sec. to Govt. Bombay, Political \& Judicial Depts.; c/o Grindlay \& Co., 54 Parliament St., S.W. 1.

1884 *Kina, Sir L. W., Kt., C.S.I., LL.D., F.S.A., I.C.S. (ret.), Craig Keigh, Aboyne, Aberdeenshire.

1921 *KISHORE, Pandit C. B., Astrologer and Astronomer, Alwar, Rajputana.

1884 *†KrTTs, Eustace John, Dudley Hotel, Hove, Sussex.

1919 Knox-Shaw, C., Waverley, Oxted, Surrey.

4601914 *Ko, Maung Ba, Extra Asst. Commissioner, Prome, Burma.

1916 *Kochar, D. R., B.Sc., Executive Engineer, Akola, Berar.

1916 *Konow, Prof. Dr. Sten, Ethongraphic Museum, Kristiania, Norway.

1906 *Krenkow, Fritz, 1 Trinity Avenue, Westcliff-on-Sea.

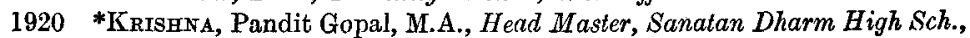
Etawah, U.P.

1921 *Krishna Rao Bhonslì, R., Rao Sahib, Stone Gift, Vepery, Madras.

1921 *KrishnaqaR, H.H. Maharaja Ksharrnish Chanda Roy Bahadur of, The Palace, Krishnagar, Nadia.

1919 *Krishnamachariar, K., B.A., Head Master, Mission High School, Vani Vihar, Tirupati, Madras.

1913 *Krishnamachariar, M., M.A., M.L., Ph.D., Bapalla, Guntur, Madras.

1922 *Krishnaswami, K., B.A., Sen. Asst. Masier, Madrasa-i-aizza, 750 Kannilal Bagh, Residency Road, Hyderabad.

4701911 *Ктом, N. J., Ph.D., Prof. of Javanese Archaeology at the University, Groenhovenstraatz, Leiden, Holland.

1909 *Kulandaiswami, R. P., Hcad Master, St. Joseph's High School, Trivandrum, Travancore.

1921 *Kunihar, Rana Hardeo Singh, Saheb Bahadur, Chief of, Kunihar State, Simla Hills.

1913 *Kunwar, Har Pratap Singh, B.Sc., 1 Turkoganj Road, Indore.

1912 * + LabBerton, Dr. D. van Hinloopen, Lecturer Indonesian Linguistics, Govt. Coll., 1 Blavatsky Park, Weltevreden, Java.

1920 *Laddu, Pandit D. K., 833 Sadashiva Peth, Poona.

1919 *LaL, Brij B., B.A., LL.B., Munsif, Phaphund, Etawah, U.P.

1904 *Lat, Hira, Rai Bahadur, B.A., Dep. Commissioner, Wardha, O.P.

1919 *Iad, Lala Mohan, M.A., Head Master, Dt. Board High School, Rahon, Jullundar, Panjab. 
1904 *Laz, Raja Madho, C.S.I., Chowkhumba, Benares.

4801910 *LaL, Shyam, M.A., LL.B., Dep. Collector, Nawaíganj, Cawnpore.

1915 *Lamb, Miss M. Antonia, 212 South 46th St., Philadelphia, Pa., U.S.A.

1902 *Land Bena, Count C., 2 Av. Désambrois, Nice, France.

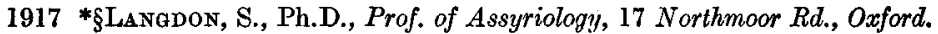

1880 Hon. 1902 Lanman, Chas. R., Prof. of Sanskrit, Harvard University, 9 Farrar St., Cambridge, Mass., U.S.A.

1911 *Ladfer, Dr. Berthold, Field Museum, Chicago, Ill., U.S.A.

1914 *IAW, Bimala C., M.A., B.A., Zamindar, 24 Sookeas St., Calcutta.

1901 *Leadbeater, Rt. Rev. C. W., 4 Raymond Rd., Neutral Bay, Sydney, Australia.

1900 *Lechmere-Oertel, Hon. Mr. F. O.

$1899 \S$ LegGe, F., F.S.A., 6 Gray's Inn Square, W.C. 1.

4901896 *Leiah, Col. H. P. P., C.I.E., Grosvenor House, Bath.

1878 *†LEPPER, C. H.

1910 *Lesný, Dr. V., Lecturer in Sanskrit, Prag University, Smichov, Zborovská, 66, Prague, Czechoslovakia.

1880 †Le Strange, Guy, 63 Panton St., Cambridge.

1921 *LeTr, H. N., D.F.C., Queen's College, Oxford.

1890 *Leveson, Henry G. A., I.C.S., 2 Edwardes Place, W. 8.

1921 *Levi, Louis, 17 Robert St., N.W. 1.

Hon. 1917 LÉvi, Sylvain, 9 Rue Guy de la Brosse, Paris.

1912 *Lenonlan, Prof. Lootfy, American Bille House, Constantinople.

1921 *Levy, R., Jesus College, Oxford.

$5001885+$ Lewis, Mrs. A. S., LL.D, Castlebrae, Cambridge.

1921 *Lewis, Rev. J. Vernon, M.A., B.D., The Manse, Brynamman, Caermarthenshire.

1897 *Lindsax, Rev. James, M.A., D.D., B.Se., Annich Lodge, Irvine, Ayrshire.

1912 *LIPSHYTz, Rev. C. T., Gorringe Park House, Mitcham, Surrey.

1879 *Lookhart, Sir J. H. Stewart, K.C.M.G., Commissioner Weihaizuei, N. China.

1919 Lovadon, Miss C. M., 124 Osmaston Rd., Derby.

1914 *广Lorimer, Maj. D. L. R., C.I.E., I.A., Political Agency, Gilg $t$, Kashmir.

1915 *Lonimer, Miss F. M. G., c/o Postmaster, Srinagar, Kashmir.

1919 Loxton, S. E., Icknield, Little Aston, nr. Sutton Coldfiell, Staffs.

1904 *LuaRd, Lt.-Col. C. Eckford, M.A., I.A., o/o Grindlay \& Co., Bombay.

5101909

*LüDers, Prof. Dr. H., 20 Sybelstr., Charlottenburg, Berlin.

1914 Lumsden, Miss Mary, c/o The Librarian, Girton College, Cambridge.

1918 *Luss, Marino M., Architect, Casa Lusy, via Lazzaretto Vecchio, No. 11, Trieste.

1910 *Macartney, C. H. H., Foxhold, Crookham, nr. Newbury, Berks.

1919 McClorn, Rev. Canon Edmund, 80 Eccleston $S q ., S . W .1$.

1900 *Maddonald, Duncan B., Theological Seminary, Hartford, Conn., U.S.A.

1921 *Macdonadd, Rev. John I., Berhampore, Ganjam.

1882*†\$Macdoneli, Arthur A., M.A., Ph.D., F.B.A., Boden Prof. of Sanskrit, Fellow of Balliol, 20 Bardwell Rd., Oxford.

1887 *McDovalt, Wm., Palmers Moor House, Iver, Bucks. 
1919 MadGragor, Rev. W., Bolehall Manor House, Tamworth.

5201919 *MacIver, Capt.David R., M.A., c/o Brown, Shipley \& Co., 123 Pall Mall, S.W. 1.

$1894 * \mathrm{M}_{\mathrm{ACLAGAN}}$, Hon. Sir E. D., K.C.I.E., C.S.I., Governor of Panjab, Lahore.

1917 *Mahajan, Suryya Prasad, Rais, Banker \& Zamindar, Murapore, Gaya, Bihar.

1921 *Mattra, Jogendra Nath, B.Sc., 58 a Colootola St., Calcutta.

1921 *Martra, Babu Ramesh C., Zamindar, Natore, Rajshahi, Bengal.

1916 *MAJid, Abdul, B.A., F.O.U., P.O. Daryabad, Bara Banki.

1921 *Ma.JUmdar, N. G., 70 Russa Rd., N. Bhawanipore, Calcutta.

1913 *Majumdar, Prof. Ramesh Chandra, M.A., Calcutta University.

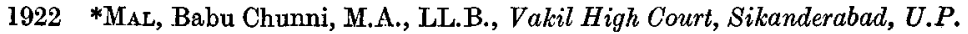

1921 *Mangar.IK, Murari Saran, B.A., Editor of "Lalita," Sevā Sadan, Meerut.

5301906

*MaNs, Fairman Rackham, Staff Surgeon, R.N., Greenlands, Nelson Rd., Sheringham, Norfolk.

1920 *MANN, Jacob, M.A.

1916 *ManuCha, Moti Lal, Oudh Commercial Bank, Ltd., Fyzabad, U.P.

1889 *\$Margonioutr, D. S., M.A., F.P.A., D.Litt., Prof. of Aratic, 88 Woodstock Rel., Oxford.

1914 *+Marielle, Madame, 1132 W. 36th Place, Los Angeles, Cal., U.S.A.

1921 *Marmorstein, Dr. A., 36 Goldhurst Terrace, N.W. 6.

1904 *Marsden, E., Ind Edic. Serv., 12 Ellerdale Rd., $N . W .3$.

1917 *Marshall, Rev. H. I., M.A., President Karen Theological Seminary, Insein, Burma.

1901 *\$MaRshalt, Sir. John, C.I.E., M.A., Litt.D., F.S.A., Director-Qen. of Archceolojy, Benmore, simla.

1914 *Martin, Rev. E. Osborn.

5401920 *Martix, Rev. John, Ingleside, Bunnington Rdl, Stockport.

1921 *Masterton-Smith, Capt P. F., M.C., Wadham College, Oxford.

1920 *Mathur, Lakshmi N., B.A., Head Master J.A.S. High Sch., Khurja, $U . P$.

1920 *Mathur, Omrao Behari, B.Sc., Naib Suba, Bhilsa, Gualior State.

1904 * Mawjee, Purshotam Vishram.

1898 *Maxwer., W. George, Carcosa, Selangor, Malay Peninsula.

1921 *Maydeli, Baron Gérard de, Ecole Nationale des Langues Orientales, 2 rue de Lille, Paris.

1921 *Mazifary, Shafi Ahmad, M.A., M.S.P., Prof. Oriental Languages, Anjuman-i-Istam, Hornby Road, Bombay.

1905 *Mazumpar, Babu Bijaya Chandra, High Court Vakil, 33/1e Lansdowne Rd., Calcutta.

1921 *Mazumdar, Babu J., Palong P.O., T'ulasar, Faridpur.

5501921 MesD, G. R. S., 9 Queen's Gate Gardens, S.W. 7.

1912 *Mead, John P., Forest Dept., Kuchin, Sarawal, via Singapore.

1907 *Meadex, Rev. H. Anderson, Barton Rectory, Nottingham.

1918 *Menzies, Capt. Rev. Jas. M., B.A., B.Sc., c/o For. Missions Bd., Presbyterian Ch. in Canada, Confederation Life Bldg., Toronto.

1919 *Mercer, Rev. Prof. S. A. B., Ph.D., D.D., 2738 Washington Boulevard. Chicago, Ill., U.S.A. 
1899 *Meston, Lord, K.C.S.I., LL.D., Hurst, Cookham Dene, Berks.

1921 MrLes, W., 21 Minster $R d .$, N.W. 2.

1921 *Mr.LS, J. P., I.C.S., c/o King, Hamilton \& Co., 4-5 Koila Ghat Street, Calcutta.

1909 *MrLne, Mrs. Leslie, c/o T. \& J. W. Barty, Csunty Bidgs., Dumblane, Scotland.

1922 *Mrngana, Prof. Alphonse, D.D., John Rylands Library, Manchester. *Mrnhas, M. Abdul Aziz, M.A., LL.B., Pleader, Gujranwala, Panjab.

*Mrsra, Pandit B. P., M.A., Executive Officer, Improvement Trust, Allahabad.

1920 *Misra, Pandit Lakshmi, B.A., Dep. Magistrate \& Coll., Patna, B. \& $O$.

1913 *Mrsra, Pandit Shyam Behari, M.A., Dep. Commissioner, Gonda.

1918 *Mrtra, Prof. Satis Chandra, B.A., Hindu Academy, Daulatpur, Khulna, Bengal.

1903 Mrrra, S. M., 6 Hayes Avenue, Queen's Park, Bournemouth, Hants.

1914 *Mrtra, Surendranath, G. T. Survey Dept., Dehradun, U.P.

1921 *Mohammad, S. Taj, M.A., M.Sc., Dy. Accountant-General, Posts and Telegraphs, Calcutta.

1882 *†Mohanlaal Visnulal Pandia, Pandit.

1922 *Mohidesv, C. S., B.A., Maulavi, Headmaster Kham-mam-meth, Warangal, Deccan.

5\%0 1919 *MomI-ud-din, Ghulam, Sufi, M.A., Supt. Normal Sch., Amraoti, Berar.

1908 *Monahas, F. J., I.C.S., Commissioner Assam Valley Dts., c/o H. S. King \& Co., 9 Pall Mall, S.W. 1.

1919 Mond, R., Coombe Bank, nr. Sevenoaks.

1912 *MookerJI, Prof. Radhakumud K., M.A., Ph.D., Vidyavaibhara, The Uninersity, Lucknou.

1916 Moreland, W. H., C.S.I., C.T.E., Bengeo Old Vicarage, Hertford.

1918 *Moreno, Prof. H. W. B., M.A., D.Litt., Central College, 12 Wellesley St., Calcutta.

1919 *Morgenstienne, Dr. Georg, Vettakollen, pr. Kristiania, Norway.

1921 *Monris, Capt. C. J., 2-3 Gurkha Rifles, c/o Grindlay \& Co., Calcutta.

1882*†\$orse, H. Ballou, LL.D., Arden, Cambertey, Surrey.

1920 *Mudaliar, D. B., Ramachandra, Supt. Govt. Printing, Rama Mandiram, Bangalore.

5801919 *Mudaliak, K. D., 11 George Sq., Edinburgh.

1920 *Mudariar, K. Narayana Swami, 75 Singannachothy St., Chintradripet, Madras.

1916 *Muhammad-SadiQ, Mufti, Prof. of Hebrew Language \& Literature, 74 Victor Av., Highland Park, Mich., U.S.A.

1921 *Muknisea, Harendra Nath, 12 Ananta Ram Mukerjea Lane, Ramkrishtopure, Howrah, Bengal.

1911 *Mukrerjea, Babu Manmatha Nath, M.A., Dep. Magistrate Collector, Chinsutra, Hooghly, Bengal.

1882 *MukerJI, Phanibhusan, Director State Education, 55 Gariahal Rd., Ballygunge, Calcutta.

1921 *Mukner.jee, Braja Lal, 12 old Post Office St., Calculta.

1916 *Mukherjee, Santosh Kumar, Vidyabhusana, Ed. "The Bansari," 52 Chaulpati Rd., Bhowanipur, Calcutta. 
1895

1921

590

\section{2}

1919

1898

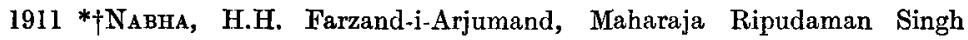

*Muller-Hess, Dr. E., Prof. of Sanskrit, Berns University, 47 Effingerstr., Berne, Switzerland.

*Muluick, A. P., 33 Nilmoney Mullick Lane, Howrah, Bengal.

*Murray, John, C.V.O., M.A., F.S.A., $50 a$ Albemarle St., W. 1.

*Mushran, Pandit Sundar N., Old Kotha Parcha St., Farrukhabad, U.P.

†Mrres, Prof. J. L., 101 Banbury Rd., Oxford.

*Mysore, Col. H. H., Maharaja Sir Sri Krishnaraja Wadiyar Bahadur, G.C.S.I., G.B.E., of, The Palace, Bangalore. Malvendra Bahadur, of, Panjab.

1921

1917

1920

1915

1918

*Nadar, Arumuga, P.K.S.A., Banker, Suakasi, India.

*Nadgowda, Shrimant N. K., Mundargi, Dharwar, Bombay.

*NaG, Abinash Chandra, B.A., B.L., Asst. Senior Judge, Gaya, Bihar.

*Nahar, Puran Chand, M.A., B.L., Zamindar, 48 Indian Mirrm St., Calcutta.

*Nardu, Mallem Chengalvarayulu, Barr.-at-Law, 18 Merchant Ss., Rangoon, Burmo.

1921

1907

1920

1900

Ext. 1910 Nasir-ul-MulK, H.E. Sir Abul Kasim Khan, G.C.M.G., c/o Persian Legation, 47 Bramhan Gdns., S.W. 5.

1920 *NaTeSA, Eli Yurkar K. G., Sastri, Vaidya Visarda, 2/30 Nachiappa Chetty St., Mylapore, Madras.

1920 *Nath, Babu Pirthi, Rais, Bhojpur, Farrukhíbid, U.P.

1922 *Natr, Pandit Surendra, M.A., LL.B., High Court Vakil, Moradabad, U.P.

1921 *NATH, Yogindra, M.A., LL.B., Vakil High Court, Ghazipore, U.P.

*Navagire, B. N., M.A., c/o Navagire \& Co., Trimbak Parasram Street, 6th Kumbharwada, Post No. 4, Bombay.

1877 Hon. 1895. Naville, Edouard, D.C.L., Prof. of Egyptology, Geneva Univ rsity; Malagny, nr. Geneva, Switzerland.

1900 *Nevilı, Col. H. R., I.C.S., C.I.E., O.B.E., Gen. Staff, Army Headquarlers, India.

1919 Newton, Miss Frances E., 156 Sloane St., S.W. 1.

1895 *Nicholson, R. A., Litt.D., 12 Harvey Rd., Cambridge.

1919 *Nicholson, Rev. Sydney, London Mission, Cuddapah, Madru.s.

HoN. 1890. Nöldeke, Prof. Theodor.

1919 *Norden, Warner M. van, 7 W. $57 t h$ St., New York, U.S.A.

1913 *Norton, E. L., I.C.S., Gorakhpur, U.P.

1920 *Nowr., V. M., Ph.D., Ed. "Social Servant," c/o Novell \& Co., Poona.

6201914

*Noyce, Frank, I.C.S., c/o Grindlay \& Co., Bombay.

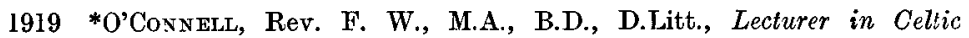
Languajes \& Literature, Queen's University, Belfast. 
1921 *OJнa, Pandit Rambhadra, M.A., LL.B., Insp. Oen. of Education, Aluar State.

1919 †OKw, A. W., 32 Denmark Villas, Hove, Sussex.

1912 *OLDhAM, J. H., M.A., Ed. International Review of Missions, 18 Succoth Av., Edinburgh.

1918 *†Ormerod, Rev. E. W., Christchurch S.P.G. Mission, Cawnpore, U.P.; 25 Upper Wimpole St., W. 1.

1917 *OsBurN, Lt.-Col. A., R.A.M.C., D.S.O., Winsley, Emsurorth, Hants.

1921 *Oulsnam, H. J., St. John's College, Cambridge.

1909 *Patra-Mall, M.D., c/o Nat. Bank of Ind., Amritsar, Panjab.

1920 *PaL, Falkirchand, Consulting Engineer, 18 Hastings St., Calcutta.

6301920 *PaL, Babu Kshitish C., B.A., "B" M.T'. Column, Accounts, Dera Ismail Khan, N.W.F.

1922 *Panalar, T. K., Tamilagam, Chintadripet, Madras.

1902 *Parasnis; Dattatraya B., Rao Bahadur, Happy Vale, Satara, Bombay.

1921 PaRBury, Miss F., 53 Egerton Gardens, S.W. 3.

1893 *\$ParatTer, F. E., M.A., I.C.S. (ret.), Vice-President, 12 Charlbury $R d .$, Oxford.

1921 *Parthar, Kunwar S. Singh, The Indian Glass Works, P.O. Firozabud, Agra, U.P.

1900 * Parla Krmed, Tbe Raja of, Ganjam, Madras.

1909 *PaRLetT, Harold G., H.B.M. Consul, Dairen, Japan.

1921 *Parmar, Deomal Damaji, Asst. Sec. Rajputana and Sindh Saini, Kshtriya Mahasabha, P.O. Khan, Sindh, J. B. Ry.

1921 *Paterson, H. M., Magdalene College, Cambridge.

6401911 *†Pattara, H.H. Maharajdhiraja Sir Bhupindar Singh, Mahindar Bahadur, G.C.I.E., G.C.S.I., T.B.E., of, Patiala State, Panjab.

1922 *PatKan, P. M., Shakespeare Hut, Keppel Street, W.C. 1.

1919 Paton, David, Univ. Library, Princeton, N.J., U.S.A.

1916 *Patterson, Lieut. W. R., Security Section (E), c/o Military Governor, Cologne, Germany.

1919 *Pی̀, Maung Tun, B.A., Lecturer, Judson College, Ra ıgoon, Burma.

1919 Penzer, Norman M., B.A., 12 Clifton Hill, N.W. 8.

1918 *Perera, Edward Walker, Member of Council Ceylon Branch R.A.S., Walavwa, Kotte, Ceylon.

1919 §Prrowne, E. S. M., F.S.A., 7 Great James St., W.C. I.

1912 *Perry, Wm. Jas., 7 York View, Pocklington, $k$. Yorks.

1905 *Petersen, T. G., Hotel Botnnique, Copenhagen, Denmark.

$6501913 * \nmid$ Pethachi Chettiak, S.R.M.C., Avl., Zamindar of Andipatti, Bishop Bungalow, Trichinopoly.

1909 *§Рнтьвт, H. St. J. B., C.I.E., I.C.S., Baghdad, Mesopotamia.

1906 *§Phllotт, Lt.-Col. D. C., M.A., Ph.D.,F.A.S.B., The Bury, Felsted, Essex.

1919 PILCher, E. J., 43 Charlwood St., S.W. 1.

1921 *PIllai, M. R. Rama Krishna, Indian Students' Hostel, Keppel Street, W.C. 1.

1919 *PIIIAY, G. Hurry K., 2 Phayre St., Rangoon, Burma.

1919 *PrLlay, N. Padmanabha, Barr.-at-Law, Karnnagapally, Travancore.

1919 Prtter, Rev. W. T., 25 De Cham Rd., St. Leonards-on-Sea. 
1911 *PrM, Alan Wm., I.C.S.

1881 § Pinches, Theophilus G., LL.D., 10 Oxford Rd., N.W.6.

1920 *Prthawalta, Manock B., Principal Parsi Virbaiji H. Sch., 20 Victoria $R d .$, Karachi.

*Prtт, St. George L. Fox, Travellers' Club, Pall Mall, S.W. 1.

*Platrs, Capt. Arnold, 65 Marshfield Rd., Chippenham, Wilts.

1921.

*Plowes, Wm. R., F.S.A., Chapel Allerton, Leeds.

1919 Plunker, Hon. Emmeline M., 19 The Grove, S.W. 10.

$1893 *$ *Plunkett, Lt.-Col. G. T., R.E., C.B., Belvedere Lodge, Sl. Mary's Rd., S.W. 19.

1920 PolfiLl, C. C., 35 Fitzroy Rd., N.W. 1.

1916 *Pope, Miss Amina Ethel, M.A., c/o The Postmaster, Shahjahanpur, U.P.

1922 Pope-Hennessy, Dane Una, D.B.E., 2 Albert Terrace, N.W. 1.

1921 *Ponov, Babu J. Saran, Zamindar, Katghar, Moradabad, U.P.

6701893 Hon. 1920 *Poussis, Louis de la Vallée, Prof. à l'Universilé de Gand, 66 Av. Molière, Uecle, Brussels.

1918 *Poynter, Capt. H. E., A.S.C., Baldwin's Canadian Steel Corporation, Ltd., Toronto, Ontario, Canada.

1909 *Prasad, Pandit Ganga, M.A., Dep. Collector, Andarkot, Meerut, U.P.

1914 *PRICE, Morgan P., Beckerstr. 24, Friedenau, Berlin.

1905 *Proctor, Henry, 146 Mallenson Rd., S.W. 12.

1913 *Prosada, Siva, Rai Bahadur, Jun. Sec., Bd. of Revenue, Allahabad, U.P.

1912 *Purser, Rev. W. C. B., M.A., St. Michael's Mission, Kemendine, Burma.

*QuRESHI, Shuraib, Brasenose College, Oxford.

1922 *Radha-Krishnan, Dr. K. M., Virudupatti, Ramnao, S. India.

1920 *Raffaeli, Saml., American Zionist Med. Unit, Jerusalem.

6801920 *Raghavan, S. Srinivasa, B.A., B.L., High Court Vakil, 33-35 Singarachari st., Triplicane, Madras.

1922 *Rahimuddin, M. Md., M.A., Prof. Osmania Univ. Coll., Kachiguda, Hyderabad, Deccan.

1921 *RAJA, C. Kunhan, B.A., c/o Barclay's Bank, Ltd., 54 Cornmarket St., Oxford.

1922 *Rajagopalaghariar, M. K., F.C.I., Public Accountant and Auditor, P. Box 1001, Kilpauk, Madras.

1922 *Rajagopalan, Mrs. M. J., B.A., Post Box 1001, Kilpauk, Madras.

1874 *†Rajanattayanuhar, H.E. Phya.

1899 *RAM, Läl Sita, F.A.U., Dep. Collector (ret.), 203 Muthiganj, Allahabxd.

1920 *Ramabeadra, Kadayam R., Sastry, B.A., Sub. Asst. Insp. of Schools, Dharmapudi, Salem, Madras.

1921 *Ramalinaam, K., Sannadhi St., Kullidaikurichi P.O., Tinnevelly.

1919 *Ramana-ŠŝAstrin, V. V., Pli.D., Vedaraniam, Tanjore.

6901909 *RamanathaN, P., B.A., Solicitor, High Court, Manonmani Vilas, Chintadripet, Madras.

1874 * †Ramasvami, Iyengar B.

1915 *Randt., Prof. Herbert Niel, M.A., I.E.S., c/o Cook \& Sons, Ludgate Circus, E.C. 4. 
1922

1869

1921

1917

1888

1921

1914

*Rarkis, J. Thomson, I.C.S. Commissioner, Darca Division, c/o Grindlay \& Co., 54 Parliament Strept, S.W. 1.

†RANsom, Edwin, 24 Ashburnham Rd., Bedford.

*Ruo, T. Raja Gopala, B.A., Ed. " S. Indian Research," Vepery, Madras.

*RAo, T. Suryanarayana, Sanskrit Coll., Kovur, Godavery Banks, Madras. *\$Rapson, E. J., M.A., Prof. of Sanskrit, 8 Mortimer Ra., Cambridge.

*Rashid, Dr. M. A., Afzulganj, Hyderabad, Deccan.

*Rawlinson, Prof. H. G., I.E.S., Principal Karnatak College, Dharwar, Bombay.

*RaT, Babu Jitendra N., B.A., Post Box 738, Calcutta.

*Ray, Jamini Bhushan Kabiraj, M.A., M.B., 46 Beadon St., Calcutta.

*RaY, Kshemada K., Smritibhusana, M.A., Zamindar, 49/1B Harish Mukerji Rd., Bhawanipur, Calcutta.

*Ray, Lalla P. N., Ramanuj Das P. C. T. Lalla's Phonetic Academy, Puri, Orissa.

*RAY, Sarat Kumar, M.A., Kumar of Dighapatiya, Dayarampur, Rajshahi, Bengal.

*Ray, Dr. Surah Chandra, L.C.H.C., Zoological Survey India, P.O. Alam Bazar, Calcutta.

Read, F. W., 65 Harley Rd., N.W. 10.

*Rees, W. H., D.D., Sch. of Oriental Studies, Finsbury Circus, E.O. 2. *Reg E, Dattatraya Vaman, B.A., Asst. Commissioner, Buldana, C.P., India.

*Remaran, Md. Jamilur, M.A., Prof. Osmania Univ. Coll., Iyderabad, Deccan.

*Rehmax, Md. Naimur, M.A., Prof. of Arabic, Persian, and Urriu, 15 Tippu Sahib Street, Mount Road, Madras.

*Reu, Pandit Bisheshwar Nath, Supt. Sardar Museum, Chandpole Gate, Jodhpur.

*Reuter, J. N., Ph.D., 21 Fabriksgatan, Helsingfors, Finland.

RICe, B. Lewis, C.I.E., Greenhalgh, Maxted Pk., Harrow.

*RIChaRds, F. J., M.A., I.C.S., c/o Binnie \& Co., Madras.

RickeTt, C. B., 27 Kendrick Rd., Reading.

*Rickmers, Mrs. W. R., Herrlichleit 5, Bremen, Germany.

$\dagger$ Riddria, Miss O. Mary, University Library, Cambridge.

* Riddina, Rev. W. Caldecott, Bradley Rectory, Ashbourne, Derbyshire.

*+RivetT-Carnac, Col. J. H., C.I.E., F.S.A., Hôtel des Trois Couronnes, Vevey, Switzerland.

1920 *R.zwr, Syed Abul H., B.A., LL.B., Asst. Income Tax Officer, Civil Lines, Aligarh, U.P.

1922 Robents, Miss H. A. E., 18 Merlon Rd., W. 8.

1910 *Robermson, Rev. Alexander, M.A., United Free Church Mission, 1 Staveley Rd., Poona.

1919 Rockstro, T. Braine, 12 Edge Hill, S.W. 19.

1921 *Rorrich, Geo. N., 1678 Massachusetts Av., Cambridge, Mass., U.S.A.

1919 Rooke, Mortimer, 54 Eccleston Square, S.W. 1.

1905 *Rose, H. A., I.C.S. (ret.), Milton House, La Haule, Jersey.

1894 §Ross, Sir Ed. Denison, Kt., C.I.E., Ph.D., Sch. of Oriental Studies, Finsbury Circus, E.C.2. 
*Ross, G. R. T., M.A., I.E.S., Prof. of Philosophy, Rangoon College, Burma.

1891 *†Rouse, W. H. D., Litt.D., Head Master, Perse School, Cambridge.

7301922 *Roy, Lt. Bijoy Prasad Singh, M.L.C., Chakdighi, Burdwan, Bengal.

1919 *Rox, Halodhur, Zamindar, 22 Bonomali Sircar's St., Calcutta.

1921 *Roy, Kumar Shib S., of Rajshahi, 26 Ballyganj Circular Rd., Calcutta.

1922 *Roy, Rai L. M. Singh, Bahadur, M.L.C., Chakdighi, Burdwan, Bengal.

1922 *Roy, Raja Mani Lall Singh, C.I.E., M.I.C., Chakdighi, Burdwan, Bengal.

1921 *Roy, Raja M. N., Chaudhury of Santosh, 1 Alipore Park Road, $E$. Alipore, Calcutta.

1916 *Roy, Srijut Jamini Kishore, M.A., Munsif, Sand:ip, Noakali, Bengal.

1921. Rubenstein, B, 5 Portland Place, $W .1$.

$1872 *+$ PU⿴囗十)

1918 §Rylands, W. Harry, F.S.A., 1 Campden Hill Place, W. 11.

740 Hov. 1887 SaChaU, Geh. Regierungsrat, Prof. Eduard, Wormser Str. 12, Berlin, $W$.

1918 *SAHA, Babu Joymongol, B.A., B.I., Asst. Head Master, K.N.H.E. School, P.O. Ujanchar, Tippera, Bengal.

1917 *SAHA, Dr. Radhika N., Chowmatha, Chinsurah, Bengal.

1918 *SAHaI, Vidyanath, Prof. Dyal Singh Coll, Lahore, Panjab.

1919 *Saksma, Debi Prasad, Sub.Dep. Insp. of Schools, Lalitpur, Jhansi, U.P.

1920 *Saksena, Ram Babu, M.A., LL.B., Dep. Collector \& Magistrate, Saharanpur, U.P.

1917 *Salariya, Sardar Gurdial Singh, Amritsar, Panjab.

1919 Sallaway, W. J. S., 49 Fellows Rl., N.W. 3.

1915 *Samaddar, Prof. Jogindranath, Patna Coll., Bankipore, B. \& $O$.

1922 Samuer, Miss M., 19 Cadogan Place, S.W. 1.

1921 *Sandapan, S., 5 Lansdowne Place, W.C. 1.

1916 *SAnYaL, Jitendranath, B.A., Zamindar, Sanyal House, Bally P.O., Howrah, Bengal.

1895 *Sarawak, H.H. Ranee of, Grey Friars, Ascot.

1891 *tSaRda, Har Bilas, B.A., Guardian to H.H. Maharawal of Jaisalmer, Ajmer.

1921 *Sarif, N., Wadham College, Oxford.

1920 *SarkaR, Prof. Subimal C., 18 Worcester Pl., Walton St., Oxford.

1908 *SarkaR, Suresa Chandra, M.A., Sub-Div. Officer, Sadar, Motihari, Bihar.

1904 *SaRtuf, Dr. Y., Ed. al-Muktataf, Cairo.

1902 †SASSOON, David S., 32 Bruton St., W.1.

1920 *Sastri, V. L., Oriental Encyclopadis Pub. Co., Box 473, Vepery, Madras.

7601850 Hon. 1906 §Satow, Rt. Hon. Sir Ernest M., G.C.M.G., Ph.D., Beaumont, Ottery St. Mary, Devon.

1922 *Sathasivam, M., Public Works Dept., Johore Bahru, India.

1919 *Satthianadian, Mrs. K., M.A., 5 Denning $R d ., N . W .3$.

1919 Sayce, Rev. A. B., Field Place, Weybridge. 
1874 †SSaYor, Rev. A. H., D.Litt., LL.D., D.D.; Hon. VIce-President, Prof. of Assyrioloyy, Queen's College Oxford; 8 Chalmers Crescent, Edinburgh.

1920 *Schomberg, Lt.-Col. R. C. F., D.S.O., 2nd Batt. Seaforth Highlanders, Meerut, India.

1905 *Schrader, F. Otto, Ph.D., Holtenauerstr. 69, Kiel, Germany.

1921 *Scotland, Patrick J., M.A., I.C.S., 17 Minard Rd., Partickhill, Glasgow.

1903 *Seddon, Charles N., Baroda, India.

1913 SeEsodia, Thakor Shri Jessrajsinghji, 17 Bury St., W.C. 1.

7901921 Sert, A., Lecturer in Arabic, Sch. of Oriental Studies, Finsbury Circus, E.C. 2.

1887 *Selt, Rev. Canon E., K.-i-H, Church Mission House, Vepery, Madras.

Hon. 1892 Senart, Émile, 18 Rue François ler, Paris.

Hon. 1913 Sermonexa, Leone Caetani, Duca di, Palazzo Sermoneta, (già Oreini) 30 Via Monte Savello, Roma.

1921

1916

*Seth, M. J., 12 Wellesley Sq., E. Calcutta.

1921

1920

1877

1920

7801922

1918

1920

1909

1921

1919

1921

1917

1922

*Sett, Madan Mohun, M.A., LI.B., Munsif, Jhansi, U.P.

Setox, Sir Malcolm, K.C.B., 13 Clarenton Rd., W. 11.

*SEwert, Cecil A. S., The Royal Page's College, Banglok, Siam.

$\S$ StwELL, R., I.C.S. (ret.), 67 Bedford Gardens, W. 8.

*SHAH, Rev. Ahmad, K.-i-H., 15/131 Civil Lines, Cawnpore, U.P.

*Sн,AH, E. Ahmad, Minu Manzil, Warris Road, Lahore.

*SHAH, Sirdar Ikbal Ali, clo Grindlay \& Co, , 54 Partiament Street, S.W.1. *Shar, Salibzada Sadiq A., c/o Dep. Commissioner, Ferozepur, Panjab.

*Shamasastry, R., B.A., Curator, Govt. Oriental Librayy, Mysore.

*Shankan, Ganga, B.A., LL.B., Munsif, Rae Barelie, Oudh.

*Sharar, Diwan Atam A., B.A., Multan, Panjab.

*Sharma, A. K., Mohindra College, Patiala.

*Sharma, Pandit Kedar Nath, Editor "Kavya-Mala", Sanghi Road, Jaipur.

ference ", Loha Mandi, P.O. Agra, U.P.

1920 *Sharma, Chaturvedi D. B., Sahityabhushana, Ed. Vaiditi Sarvasva, Daraganj, Allahabad, U.P.

7901919

1920

1922

1921

*Sharmay, Dr. B. D., B.A., 25a Madan Boral Lane, Calcutia.

*SHastri, Mangal D., M.A., Mohalla Sahukara, Budaun City, U.P.

*Shelley-Thompson, A. J., 8D Change Alley, Singapore.

1912

*Sherman, Mangi Lall, B.A., I.L.B., Foreign Sec. to Orchha Durbar, Tikamgarh, Orchha State, $C$. India.

1921

1921

1914

1919

1921

*Sherrate, Rev. W., Brit. \& Foreign Bible Society, Rangoon, Burma.

*SherwaNt, Haroon Khan, B.A., Barr.-at-Law, Osmania University College, Hyderabad, Deccan.

*Sirrofr, L. Framroz, B.A., B.F., 34 Westbourne Road, N. 7.

*ShutTleworth, H. L., I.C.S., 36 Lambolle Road, N.W. 3.

Srbrex, Ernest, 48 Manor Park, Redland, Bristol.

*Siddiq1, A., M.A., Ph.D., Prof. of Arabic, Principal's Office, Osmania Univ. Coll., Hyderabad, Deccan.

1920 *SIDERSky, Michel, B.A., Queen's College, Oxford. 
1910 *Stmpson, Mrs. Alicia Hall, c/o Sir C. R. McGrigor \& Co., 39 Panton St., W.C. 2.

1914 *Srmpson, Rev. D. C., M.A., Manchester College, Oxford.

1922

1907

1914

*Sinc H, Hargian, Headmaster D.A.V. Sch ol, Bulandshahr, U.P.

*Srngh, Kahan, Sirdar of Nabha, P.O. Mandi Phul, via Bhatinda, N.W. Railway.

*Snngh, Lala Gulbahar, M.A., LL.B., Prof. of Sanskrit, Govt. College, Lahore, Panjab.

$1902{ }^{*}+$ Singh, Raja Pertab Bahadur Singh, C.J.E., of Tiraul, Partabgarh, U.P.

1917 *Singr, Sahibzada Bhagat Lakshman, Dist. Inspector of Schools, Ludhiana, India.

1920 *Srngh, Sahibzada Sher, M.Sc., Asst. Div. Forest Off., Jammu \& Kashmir State.

8101895 *†Sinha, Kunwar K. Pal, Raio Kolla, P.O. Narki, Agra, U.P.

1921 *Sinha, Prof. M., B.A., 30 Park St., Calcutta.

1920 *Sinha, Babu Manoranjan, Asst. Settlement Officer, Chupra, B. \& O.

1913 *Sinha, Babu Rudra Datta, M.A., LL.B., Vakil, High Court, Lucknow, U.P.

1921

*Sinha, Kumar Gangananda, B.A., M.A., Srinagar P.O., Purnea, $B . \& O$.

1920

1920

1920

1921

1900

*Sircar, Babu Ganopati, Vidyaratna, 69 Beliaghatta Main Rd., Calcutta.

*Strkar, P., M.A., 180 Up. Circular Rd., Calcutta.

*Sitaram, K. N., B.A., 21 Cromwell Road, S.W. 7.

*Sitaraman, Kadayam R., B.A., B.L., High Court Vakil, 8/2 Badriah Garden Street, Park Town, Madras.

1921 *Smant, J. E., B.A., M.C., C.M.G., 34 Albemarle Av., High West Jesmond, Newcastle-on-Tyne.

1922 *Sмiтн, F. Trednell, M.A., B.D., Oriel College, Oxford.

Hon. 1909 Snouck Huraronje, Prof. C., Rapenburg 61, Leiden, Holland.

1907 Soane, Maj. E. B., C.B.E., c/o H. S. King \& Co., 9 Pall Mall, S.W. 1.

1915 *Som, Atul Chandra, B.A., Land Acquisition Officer, Chaibasa P.O., Singbhum.

1920

*Sonper, H.H. Maharaja Sri Sir Bir Mitrodaya Singh Deo, Dharmanidhi, K.C.I.E., of, Sonpur Feudatory State, Sonpur Raj P.O., via Sambalpur.

1912 *Sood, Babu Hira Lal, Sub.Judge, Kotah, Rajputana.

1920 *Sootnill, W E., M.A., Prof. of Chinese, 11 Norham Rd., Oxford.

1922 SPINk, Capt. Harold H. M., A.M.I.C.E., Slaney Cottage, Spellhurst, Kent.

1910 *Spooner, D. B., Ph.D., Asst. Director Gen. Arch. Survey, Benmore, Simla.

1908 *Spoonze, Mrs. D. B., Elysium Hotel, Simla.

1918 *Srikantaiya, S., B.A., B.L., Bangalote, Madras.

1920 *Srinivasadeari, C. S., M.A., Prof. of History, Pachaiyappa's College, Madras.

1910 *Stä̈L-Holstein, Baron A. von, c/o Peking Club, Peking, China.

1919 *Stanton, J., High St., Chorley, Lancs.

1907 *Stapleton, H. E., I.E.S., c/o Director Public Instruction in Bengal, Writers' Buildings, Calcutta. 
1909 *StaRk, Herbert A., Additional Asst. Director Public Instruction Bengal, Writers' Buildings, Calcutta.

1921 *Stavrides, G. Paniotes, Royal Societies' Club, St. James's St., S.W. 1.

1887 *\$Stein, Sir Aurel, K.C.I.E., Ph.D., D.Litt., D.Sc,, Supt. Ind. Arch. Survey, c/o Postmaster, Srinagar, Kashmir.

1905 *Stevens, George F. A., Sultanabal, Peraia.

8401921 *Stevenson, W. B., D.Litt., Prof. of Hebrew \& Semitic Languages, 7 College Ccurt, The University, Glasgow.

1912 *Stewart, J. A., I.C.S., c/o T. Cook \& Son, Rangoon, Burma.

1915 Storey, C. A., The Library, India Ofice, S.W. 1.

1921 *Stowkl, F. de la M., Malay Educational Service, The Malay Coll., Kuala Kangsar, Peral, F.M.S.

1912 *Strauss, Dr. Otto, Die Universitët, Kiel, Germany.

1919 Stringer, E. W.

1918 *Surrahmanya, Palamadai P., Sastri, B.A., Cathedral P.O., Madras.

1921 *Subramanyam, M., M.B., Health Officer, Ootacamund, Madras.

1917 *Suryanarayana, Satalar S., Lalagraharam, Madura.

1893 *+Svasti Sobhana, H.R.H. Prince, Bangkok, Siam.

8501921 *Swinson, R. F. G., B.A., St. Stephen's House, Oxford.

1895*†SYxes, Brig.-Gen. Sir Percy Molesworth, K.C.I.F., C.B., C.M.G.

1910 *Tabard, Rev. Father A. M., M.A., M.B.E., K.-i.-H., The Cathedrat, Shoolay, Bangalore.

1921 *TagGaRT, W. Q., I.C.S., clo The Secretariat, Rangnon, Burma.

1915 *Tagore, Babu Kshitendra Nath, B.A., 6/1 Dwarkanath Tagore Lane, E. Gate, Jorasanko, Calcutta.

1922 *TAgORe, Kshitish Chandra, M.V.R.S., Hon. Magistrate and Zemindar, P.O. Pakuria, Rajshahi, Bengal.

1912 *Tahoon, Gholam, Interpreter, High Conrt, $44 b$ Police Hospital Rd., Calcutta.

1896 *TaKakUsu, Jyan, Ph.D., 902 Sendagaya, Tokyo, Japan.

1897 *Talbot, Walter Stanley, C.I.E., c/o H. S. King \& Co., 9 Pall Mall, S.W. 1.

Hon. 1910 Taltevist, K. L., Prof. of Oriental Literatures, Fabriksgasse 21, Helsingfors, Finland.

8601921 *TAмre, G. C., B.A., LL.B., Sec. to Maharashtrya Ma zakosh M. Ltd., Conncil Hall, Nagpur, C.P.

1913 *Tampyah, T. Isaac, Barr.-at-Law, 18 Beach St., Penang, Malay Peninsula.

1921 *Tamr, A. Parameswaran, Indian Students' Hostel, Keppel Street, W.C. 1.

1914 *fTampi, Vatasseri Sri Velayudhan, son of H.H. Maharaja of Travancore, Trivandrum, Travancore.

1921 *T'ANgri, Rustom H., 72 Elgin Crescent, W.11.

1912 *Tannax, Mohan Lall, Barr.-at-Law, Sydenham College Hostel, Chami $R d .$, Bombay, 4.

1916 *Tanmarativa, Nutu G., Lalgola P.O., Murshidabad, Bengal.

1897 * T. Ate, George P., Ind. Survey Dept. St. Quentin, Naini Tal, U.P.

1893 *Taw Sern Ko, C.I.E., K.-H.H., I.S.O., Peking Lodge, West Moat Rd., Mandalay, Burma. 
1911 *†Teape, Rev. Wm. Marshall, M.A., South Hylton Vicarage, Sunderland. 8701879 *\$Temple, Lt.-Col. Sir Richard C., Bart., C.B., C.I.E., F.S.A., Director, The Nash, Worcester.

1922 *Tennant, Hon. Mrs. Ruth, St. Anne's Manor, Sutton, Loughborough.

1898 *Thatcher, Rev. G. W., M.A., Camden College, Sydney, N.S.W., Australia.

$1921{ }^{*}$ Thirtur, Rev. David G., 23 Borthwick Rd., E. 15.

1905 *Thintle, James Wm., LI.D., 23 Borthwick Rd., E. 15.

1917 *Thomas, E. J., M.A., Under Librarian, University Library, Cambridge.

1898 §Thomas, F.W., Ph.D., Hon. SeCretary, Librarian, India Office, S.W. 1.

1918 Trompson, Sir Herbert, Bart, The Old House, Apsley Guise, Benls.

1907 *Thompson, J. Perronet, I.C.S., c/o Grindlay \& Co., 54 Parliament St., S.W. 1 .

1919 *\$Thompson, R. Campbell, M.A., F.S.A., Milburn Lodge, Boar"s Hill, Oxford.

880 Hon. 1909 Thomsen, Prof. Dr. Vilhelm, St. Knuds Vej 36, Copenhagen, Denmark.

1899 †\$Tномson, H. Lyon, F.S.A., 34 St. James's Street, S.W. 1.

1880 * † Torburn, S. S., Bracknell House, Bracknell, Berks.

1912 *Thornton, H. A., Supt. N. Shan States, Lashio, Burma.

1922 Trckel工, E. Templer, A.M.I.C.E., 5 The Common, S.E. 16.

1903 *Tilbe, Rev. H. H., Ph.D., Amer. Baptist Mission, Rivevview, Ahlone, Burma.

1921 *Trn, Pè Maung, Exeter College, Oxford.

1921 *Tor.kowskr, S., 3 Allenby Road, Tel-Aviv, Jaffa, Palestine.

1895 * Travancore, H.H. Sri Padmanabha Sir Rama Varma Maharaja Raja Bahadur, G.C.S.I., G.C.I.F., of.

1921 *Tkench, C. G. C., I.C.S., Nagpur, C.P.

8901917 *Tripatur, Prof. Deva Datta, Sahityacharya, Patna College, Bankipore, $B, \& O$.

1918

*TeIPater, Pandit Ram P., Reader in Modern Ind. Hist., The University, Allahabad, U.P.

1921 *Trollope, Rt. Rev. Mark N., Bishop in Corea, Eng. Ch. Misision, Seoul, Corea, via Japan.

1921 *Tвотт, A. C., H.B.M.'s Legation, Teleran, c/o The Foreign Office, S.W. 1.

1912 *Troup, James, "Woolescote," Spring Hill, Ventnor, I.W.

1919 *Trumper, Lt.-Commander V. I., R.N.R. (ret.), Hon. Sec. Palestine F.cp. Fund, Maison Perrin, Port Said.

1902 *Tsain, Moung, Pegu, Lower Burma.

1900 *Tuckweld, Rev. John, 1 Onslow Gdns., N. 10.

1912 *Turner, Prof. R. L., Sigrā, Benares, $L . P$.

1919 *Turner, Capt. Vere E., c/o Thomson, Lehzen \& Co., Ltd., Chartered Banl Buildings, Calcutta.

5001882 *UnaIPUR-Mewar, H.H. Maharajdhiraja Maharana Sir Fateh Singh, Bahadur, G.C.S.I., G.C.I.E., G.C.V.O., Maharana of, Rajputana.

1919 *Ur, Prof. H., c/o Sōtöshū Daigaku Komanzawa Mura, Tolyo-fu, Japan.

1920 *URDhWARESHE, Waman Gopalrao, M.A., Kävya-Tirtha, 35 Krishnapura, Indore City, Cent. India. 
1921

1919

1922

1921

1922

1920

1921

9101921

1921

1921

1884

1913

1921

1922

1905

$\mathrm{I} 899$

1908

1914

1912

1919

1912

1921

1907

1912

1919

1921

930

1916

1914

1907

1900

1921

1921

1921

1921

1906

1921

*Vadngirar, V. V., 44 Hilldrop Road, N. 7.

*VaIshy a, Ramji D., F.R.S.A., Sweet Cottage, Gwalior.

Valvarise, Hugo, Sec. to the Finnish Legation, 2 Moreton Gardens, S.W.5.

*Vartast Sattir, Ma'sud Ali, Asst. Director of Public Instruction, Bhopal State, O. India.

*VARMA, B. Brij, M.L., B.A., L.T., Headmaster D.A.V. High School, Agra, U.P.

* VarMa, Gopi N. S., Zamindar, Mohalla Quanungoyam, Bereilly, U.P.

*Varma, Kunwar G. Suryabhan, Editor "Kshtriyamali Saini Mittra" P.O. Kuram, Akola, C.P.

*Varma, L. A. Ravi, Ophthalmic Hospital, Trivandrum.

*VARMa, Prem Mohan Lal, B.Sc., of "Gokul Nivas ", Budaun, U.P.

*Varma, Sheo Prasad, M.A., Prof. of English, Robertion Coll., Jubbulpore.

*+VASUDEv, Mãdhav Samarth, R. R., B.A.

*Vidya äbũ̃sana, Pandit Upendranath, B.A., Sr. Prof. of Sanskrit, City College, Calcutta.

*VIran, S. S. Guana, c/o Nat. Bank of India, 26 Bishopsgate, E.C.2.

*Viverananda, S., Indian Telegraphs, Madras.

*Voger, Prof. J. Ph., Ph.D., The University, Leiden, Holland.

Vost, Lt.-Col. W., I.M.S., 26 Crystal Palace Pk. Rd., S.E. 26.

*Wackervager, Prot. Dr. Jakob, University of Basle, Switzerland.

WADDELL, Lt.-Col. L. A., C.B., C.I.E., LL.D., I.M.S., c/o H. S. King \& Co., 9 Pall Mall, S.W. 1

*Wahid, Maulvi Syed Abdul, M.A., Prof. Mayo College, Ajmer.

*Waushat, Raza Ali, Sahib-i-Diwan, 2/1/2 Dillusha St., P.O. Bally. gunge, Calcutta.

WaIte, Mrs. A. R., 8 Mount Park Crescent, W. 5.

*†WALk Gr, Rev. C. T. Harley, M.A., 28 Warnboyongh Ru, Oxford.

Wallace, G. Malcolin, 31 Holmwood Rd., Bromley, Kent.

*Watsh, E. H. C., OS.I., I.C.S., Membey Bd. of Revenue, Bankipur, 14 Linton $R d$., Oxford.

*War Ofrion. Officer in Charge, Far Eastem Sub-section, Whitehall, S.W. 1.

Warburton, Rev. B. A., 90 Billinge Rd., Spring Bank, Wigan.

*Ward, Lt. J. F., Selwyn College, Cambridge.

*Wardrop, Oliver, C.M.G., M.A., Brit. Consulate Gen., 27 Rue Erckmann. Chatrian, Strasbourg, France.

*Warming, Gen. P. L. E., Bangkok, Siam.

*Watson, H. D., I.C.S. (ret.), Upper Gates, Ryder Hill, Guildford.

*WeIR, T. H., B.D., Rockcliff, Bowling, Dumbartonshire.

WeLd-BuUndel., H., 17 Ovington Sit, S.W. 3.

*Wentwakar, Rainchandra N., Wadham College, Oxford.

*Wernham, J. W. K., M.C., Wadham College, Oxford.

*Whstraxi, A. R. C., I.C.S., c/o Chief Bec. to Govt. of Madras.

*Whiteinead, R. B., I.C.S., Lahore

WhyMaNT, A. N. J, 90 Thornton Av., W. 4.

1919 *Wлекнам, H. T., Ind. Police, Peshawar, N.W.F.P. 
1899 *Wickremasingne, Don M. de Zilva, Sih. of Oriental Studies, Finsbury Civcus, E.C. 2.

1911 *Wisewardene, Don Richard, Rickman House, Colpetty, Colombo, Ceylon.

1916 *WILBerforen-Belt, Maj. H., Asst. Resident, Aden, Arabia.

1913

*Wilder, Rev. G. A., M.A., D.D., Chicore Mission Sta., Chipinga P.O., Melsetter Dt., Rhodesia, S. Africa.

1921 *Wit.iams, Capt. B. T., Sevenoalss School, Sevenoalis, Kent.

1921 *Wrinams, Eric T., Colonial Secretariat, Singapore, Straits Settlement.

1915 *Wrulams, L. F. Rushbrook, B.A., B.Litt., O.B.E., c/o Home Dept., Govt. of India, Simla.

1919 WiLlis, G. de I., Leconfield Estate Off., Ennie, Co. Clare.

1921 Willotghey-Meade, G., c/o N. Brit. \& Mercantile Ins. Co., Ltd., 60 Watling St., E.C. 4.

9501919 *WrNckwoRTr, Chauncey, fritzwilliam Hall, Cambridge.

1912 *Winstent, R. O., D.Litt., Singapore, Straits Settlement.

1896 *Woon, J. Elmsley, 4 Glenisla Gdns., Erlinburgh.

1907 *Woodley, Rev. Edward C., American Mission Seminary, Marash, Turkey in Asia.

1919 *Woold.my, Capt. C. Leonard, R.F.A., Uplands, Bathrick Hill, Bath.

1909 *Woods, Prof. Jas. H., Ph.D., Harvard University, 179 Brattle St., Cam. bridge, Mass., U.S.A.

1906 *Woolser, A. C., M.A., 11 Racecourse Rd., Lahare.

1900 *Wonkman, Mrs. Bullock, clo Amer. Exp. Co., 6 Haymarket, S. IF. 1.

1894 *WRI $x$ HT, H. Nelson, I.C.S., Judges' House, Bareilly, U.P.

1920 *WYNCe, Lionel M., C.I.E., C.B.E., I.C.S. (ret), Pine Hill, Camberley, Surrey.

$9601919{ }^{*}$ WYNTER, A. E., M.D., M.R.C.S., L.R.C.P., 26 Oakfield Rd, Ciifton, Bristol.

1919 *Y YHUDA, Dr. A. \$., 45 Brondeshury Rd., N.W. 6.

1911 *Yazdani Mas'Ud, Ghulam, Supt. of Arch., Nizam's Dominions, Hyderabad, Deccan.

1921 *YeuuIn, David, M.B.E., Sicron Moste, Jevusalem.

1921 *YerTs, Maj. L. M., M.C., c/o Civil Commissioner, Baghdad, Mesopotamia.

1910 §YसrTs, W. Perceval, O.B.E., Jr. United Service Club, Charles St., S.W. 1.

1913 *Yusoor, S. Azhar, Yusoof Manzil, 53/82 Pitar Kunda, Benarns City.

1921 *Yusur, Md., M.A., Head Master, Calcutta Madrasah, Cxlcutta.

1895 Yusur-ALI, Abdullah ibn, C.B.E., I.C.S., M.A., LL.M., cio King, King \& Co., Bombay.

1913 *Zimmermann, Rev. Robert, S.J., St. Xavier's Coll., Cruickshank Rd., Bombay.

9701920 *Zutsnt, Pandit Chand N., Hapry Cottage, Morar, Gwalior State.

1922 Zutsir, Lambodhar, 21 Cromwell Roud, S.W. 7. 


\section{Wonorary sidembers}

1906 Professor Réné Basset, Algiers.

1885 Sir Ramkrishna Gopal Bhandarkar, K.C.I.E., C.S.I., Poona, Bombay.

1920 Professor W. Caland, Utrecht.

1919 Rt. Rev. L. C. Casartelli, Bishop of Salford.

1920 Professor C. Clermont-Ganneau, Paris.

1893 Professor Henri Cordier, Paris.

1908 Professor Freidrich Delitzsch, Berlin.

1918 Monsieur A. Foucher, Paris.

1921 Mahamahopadlayaya T. Ganapati Sastri.

1893 Professor Ignaz Goldziher, Budapest.

1898 Professor Ignace Guidi, Rome.

1921 Mahamahopadhyaya Haraprasad Shastri, C.I.E., M.A.

1902 Professor Houtsma, Utrecht.

1912 Professor Hermann Jacobi, Bonn.

1904 Professor Julius Jolly, Wurzburg.

1902 Professor Lanman, Cambrifge, U.S.A.

1916 Professor Sylvain Lévi, Paris.

1895 Professor Edouard Naville, Geneva.

1920 Professor Theodor Nöldeke, Karlsruhe.

1920 Professor L. de la Vallée Poussin, Brussels.

1887 Professor Eduard Sachau, Berlin.

1906 Sir Ernest Satow, G.C.M.G.

1892 Monsieur Émile Senart, Paris.

1913 Leone Caetani, Duca di Sermoneta, Rome.

1909 Professor C. Snouck Hurgronje, Leiden.

1910 Professor K. L. Tallqvist, Helsingfors.

1909 Professor Vilhelm Thomsen, Copenhagen.

\section{Extraordinary IDember}

1910 H.E. Sir Abul Kasim Khan, G.C.M.G., Nasir-ul-Mull.

Note.-The number of Honorary Members is limited by Rule 9 to thirty.

\section{Gold Sibedallists}

$\begin{array}{ll}\text { N.B. - The Gold Medal was founded in } 1897 . \\ 1897 & \text { Professor E. B. Cowell: } \\ 1900 & \text { E. W. West. } \\ 1903 & \text { Sir William Muir. } \\ 1906 & \text { G. U. Pope. } \\ 1909 & \text { G. A. Grierson. } \\ 1912 & \text { J. F. Fleet. } \\ 1915 & \text { Arr. Agnes Smith Lewis. } \\ 1918 & \text { V. A. Smith. } \\ 1922 & \text { Professor H. A. Giles. }\end{array}$




\section{JBraltch allo $\mathcal{E}$ ssociate societies}

The Bombay Branch of the R.A.S.

The Ceylon Branch of the R.A.S.

The North China Branch of the R.A.S.

The Korean Branch of the R.A.S.

The Madras Literary Society and Auxiliary of the Royal Asiatic Society. The McGill University Oriental Society, Montreal.

The Straits Branch of the R.A.S.

The Asiatic Society of Bengal. 


\section{LIST OF LIBRARIES AND NON-MEMBERS}

\section{SUBSCRIBING TO THE \\ JOURNAL OF THE ROYAL ASIATIC SOCIETY}

Aberdeen : University Library.

Aberystwyth : University of Wales. Adelaide : Public Library.

Adyar Library, Madras.

Ajmer : Mayo College.

Aligarh : Lytton Library, M.A.O. College.

Allahabad : Public Library.

Allahabad University : History Department.

Allahabad: Muir Central College.

Baltimore: Johns Hopkins University Library.

Baltimore: Peabody Institute.

Bangalore: Director Archæological Researches.

Bangalore: Inspector - General of Education.

Bangkok : Juibrary.

Bangkok : Vajirañãna National Library.

Bankipur : Patna College

Beirut : Syrian Protestant College.

Benares: Government Sanskrit

Library.

Benares: Hindu University.

Benares : Queen's College.

Berkeley : California University

Library.

Berkeley, U.S.A. : Pacific School of Religion.

Bhavanagar: Samaldas College.

Birmingham : Public Library.

Bombay : Elphinstone College.

Bombay : Jamjetsu N. Petit. Institute.

Bombay : University Library.

Boston, U.S.A. : Museum of Fine Arts.

Brighton : Public Library.

Bristol University.

Bryn Mawr : College Library, Penn., U.S.A.

Cairo: Institut Français.

Cairo: Ministry of Education.
Cairo: Sultania Training College.

Calcutta : Bangiya Sahitya Parishad Mandar:

Calcutta : Imperial Library.

Calcutta : Indian Museum, Archæological Section.

Calcutta : Madrasah.

Calcutta : Presidency College.

Calcutta: Ripon College.

Calcutta : Sanskrit College.

Calcutta : Scottish Churches' College.

Calcutta : St. Xavier's College.

Calcutta : University Library.

Cambridge: Havard College.

Central Provinces : Chief Commissioner.

Chester, U.S.A. : Bucknell Library.

Chicago : Newberry Library.

Chicago: University Library.

Chittagong: The College.

Cincinnati: Public Library.

Cleveland : Adelbert College Library.

Constantinople : Rabert College.

Copenhagen: Royal Library.

Copenhagen : University Library.

Dacca : Jugannath College.

Dairen, Manchuria : Bank of Chosen.

Detroit : Public Library.

Dharwar : Karnatak College.

Duntroon, Australia : Royal Military College.

Edinburgh: Public Library.

Edinburgh : Royal Scottish Museum.

Egmore, Madras : University Library.

Florence: Biblioteca Nazionale.

Gauhati : Cotton College.

Geneva: Bibliothèque Publique.

Geneva : League of Nations.

Glasgow : Mitchell Library.

Goteborg, Sweden : Librario Wittergren.

Gottingen: Universitäts Bibliothek.

Gwalior State: Inspector of Archæology. 
Hankow: Hankow Club.

Haverford, U.S.A. : College Library.

Hosokawa, M., Esq., Tokyo.

Hyderabad : Nizam's College.

Hyderabad: Nizam's State Library.

Hyderabad: Osmania University College.

Indianapolis, U.S.A. : College of Missions,

Ishihama, J., Esq., Osaka.

Ithaca : Cornell University Library.

Jingu Kogakukwan, Japan.

Junagadh Archæological Society, Kathiawar.

Kanazawa, Japan : Fourth High School.

Khartoum : Director of Education.

Kyoto: Indian Philosophy.

Lahore: Dayanand Anglo-Vedic College.

Lahore: Dyal Singh Library Trust

Lahore: Forman Christian College.

Lahore: Panjab Public Library

Lahore: Panjab University.

Lahore : Tilak School of Politics.

Liverpool: The Athenæum.

Liverpool : Institute of Archæology.

London: Science Iibrary.

Athenæum Club.

British Museum.

East India United Service Cinb.

London Library.

Louisville, U.S.A. : S. Baptist Theological Seminary.

Lucknow : Canning College.

Lucknow : Provincial Museum.

Lucknow : Public Library.

Lund : Kungl. Universitets Biblioteket.

Lyon: University Library.

Madras: Archæological Survey.

Madras : Connemara Public Library.

Madras : Kumbakonam College.

Madras : National College of Commerce.

Madras : Oriental Manuscripts Library.

Madras : Presidency College.

Manchester: Free Reference Library.
Manchester: John Rylands Library.

Manchester : Lancashire Independent College.

Manchester University (Victoria).

Manchuria : "Chosabu."

Manila : Bureau of Science.

Maynooth : St. Patrick's College.

Meadville, U.S.A. : Alleghany College Library.

Melbourne: Victoria Public Library.

Michigan University.

Minneapolis Athenæum.

Montreal : McGill University.

Muzaffarpur : Greer Bhumihar Brahman College.

Mysore Archæological Researches Office.

Mysore: Government Oriental Library.

Mysore: University Library.

Nanking: National South-Eastern University.

Newcastle-on-Tyne: Public Library.

New York: Metropolitan Museum of Art.

New York: Jewish Theological Seminary.

New York: Missionary Research Library.

New York: Public Library.

New York: Union Theological Seminary.

Niigata: Koto-Gakko.

Ottawa : Library of Parliament.

Oxford: Indian Institute.

Paris : Institut de France.

Patna: B. \& O. Research Society.

Pavia: Facotta di Lettere-e-Jilosofia.

Peshawar: Archæological Survey, Frontier Circle.

Philadelphia : Commercial Museum.

Philadelphia Library Company.

Pittsburg : Carnegie Library.

Pittsburg : Western Theological Seminary.

Poona : Archæological Survey, Western Circle.

Poona: Fergusson College.

Princeton: Theological Seminary.

Princeton University Library. 
Santiniketan : $\quad$ Brahmacharya- Tokyo : Institute of History, Imperial Ashrama.

University.

Seattle: University of Washington. Tokyo: Dr. G. E. Morrison Library.

Simla : Bureau of Education.

Tokyo: Noshomu.

Srinagar : Sri Pratap Singh Museum. Tokyo: Shūkyo-daigaku Library.

Stationary Office.

Toronto Reference Library.

Stockholm : Nordiska Bokhandel. Trichinopoly : St. Joseph's College.

Stockholm : Royal Library.

Strasbourg: Bibliothèque Utrecht: University Library. versitaire et Regionale.

Suga, Mr. T.

Sylhet: Murarichand College.

Tinnevelly: Hindu College.

Uni. Valkenberg: Ignatius College.

Vizianagram : Maharajah's Sanskrit College.

Winnipeg: University of Manitoba. Zurich : Bibliothèque Centrale.

Note.-There are other libraries which subscribe through the booksellers. The Secretary would be much obliged by the Librarians of such libraries sending their names to be added to the above list.

\begin{tabular}{|c|c|c|c|c|}
\hline \multicolumn{5}{|c|}{ SUMMARY } \\
\hline & & & June $1,1921$. & June 1, 1922. \\
\hline Resident Members & . & • & 153 & 135 \\
\hline Resident Compounders . & - & • & 8 & 13 \\
\hline Non-resident Members . & - & • & 632 & 737 \\
\hline Non-resident Compounders & . & • & 62 & 64 \\
\hline Library Members . & $\cdot$ & - & 1 & 1 \\
\hline \multirow[t]{2}{*}{ Honorary and Extraordinary } & \multirow{2}{*}{\multicolumn{2}{|c|}{ Members }} & 27 & 27 \\
\hline & & & 883 & 971 \\
\hline \multirow{2}{*}{$\begin{array}{r}\text { Subscribing Libraries, etc. } \\
\text { Total }\end{array}$} & - & • & 169 & 170 \\
\hline & - & - & 1052 & 1141 \\
\hline
\end{tabular}




\section{ROYAL ASIATIC SOCIETY PUBLICATIONS}

ASIATIC SOCIETY MONOGRAPHS. Vol. XVIII. Linguistic Studies from the Himalayas. Being Studies in the Grammar of fifteen Himalayan Dialects, by the Rev. T. GRAHAME BAILEY, D.Litt., B.D., M.A. 1920.

\section{PRIZE PUBLICATION FUND. Vol. v.}

Ishkashmi, Zebaki, and Yazghulami. An account of three Eranian Dialects, by Sir GEORGE GRIERSON, K.C.I.E., Ph.D., D.Litt., LL.D., F.B.A. 1920.

\section{Vol. VI.}

The Phonology of the Bakhtiari Badakshani and Madaglashti Dialects of Modern Persian.

By Major D. L. R. LORIMER, C.I.E.

ORIENTAL TRANSLATION FUND, Vol. XXVII. The Table-talk of a Mesopotamian Judge. ARABIC TEXT.

Being the first part of the Nishwār al-Muhādạahah or Jāmi‘ al-Tawarikh of Abu 'Alī al-Muhassin al-Tanükhi. Edited from the Faus MS. by D. S. MARGOLIOUTH, D.Litt., F.B.A.

IN THE PRESS.

\section{ORIENTAL TRANSLATION FUND.}

El-Asatir. By M. GASTER, Ph.D.

Translation of Table-talk of a Mesopotamian Judge. Nishwār al-Muhädarah or Jāmi' al-Tawārikh of Abu 'Alī al-Muhassin al-Tanükhi. By D.S.MARGOLIOUTH, D.Litt.,F.B.A. 


\section{ROYAL ASIATIC SOCIETY \\ OF \\ GREAT BRITAIN AND IRELAND. \\ 74 Grosvenor Street, W. 1 .}

\section{SPECIAL NOTICE.}

The ROYAL ASIATIC SOCIETY publishes Quarterly an Illustrated Journal, containing Original Articles on the Languages, the Archæology, the History, the Beliefs, and the Customs of the East.

Each quarter the principal books published on Oriental subjects are reviewed.

The Annual Subscription to the Society is THREE GUINEAS a Year for Resident, and THIRTY SHILLINGS a Year for Non-Resident, Members.

Each Member who has paid his Subscription for the current year receives the Journal post free, has the use of the Library at the Society's rooms, and admission to the meetings of the Society. Three-guinea Subscribers may also borrow books.

Libraries and Non-Members may obtain the Journal post free by a Subscription of Two Pounds Eight Shillings a year if paid in advance direct to the Secretary. The price of each par. separately is Fifteen Shillings.

NOTICE TO MEMBERS AND STUDENTS.

Tea can always be supplied by the Caretaker at a small charge. 